Prepared in cooperation with the U.S. Fish and Wildlife Service

\title{
Neosho Madtom and Other Ictalurid Populations in Relation to Hydrologic Characteristics of an Impounded Midwestern Warmwater Stream: Update
}

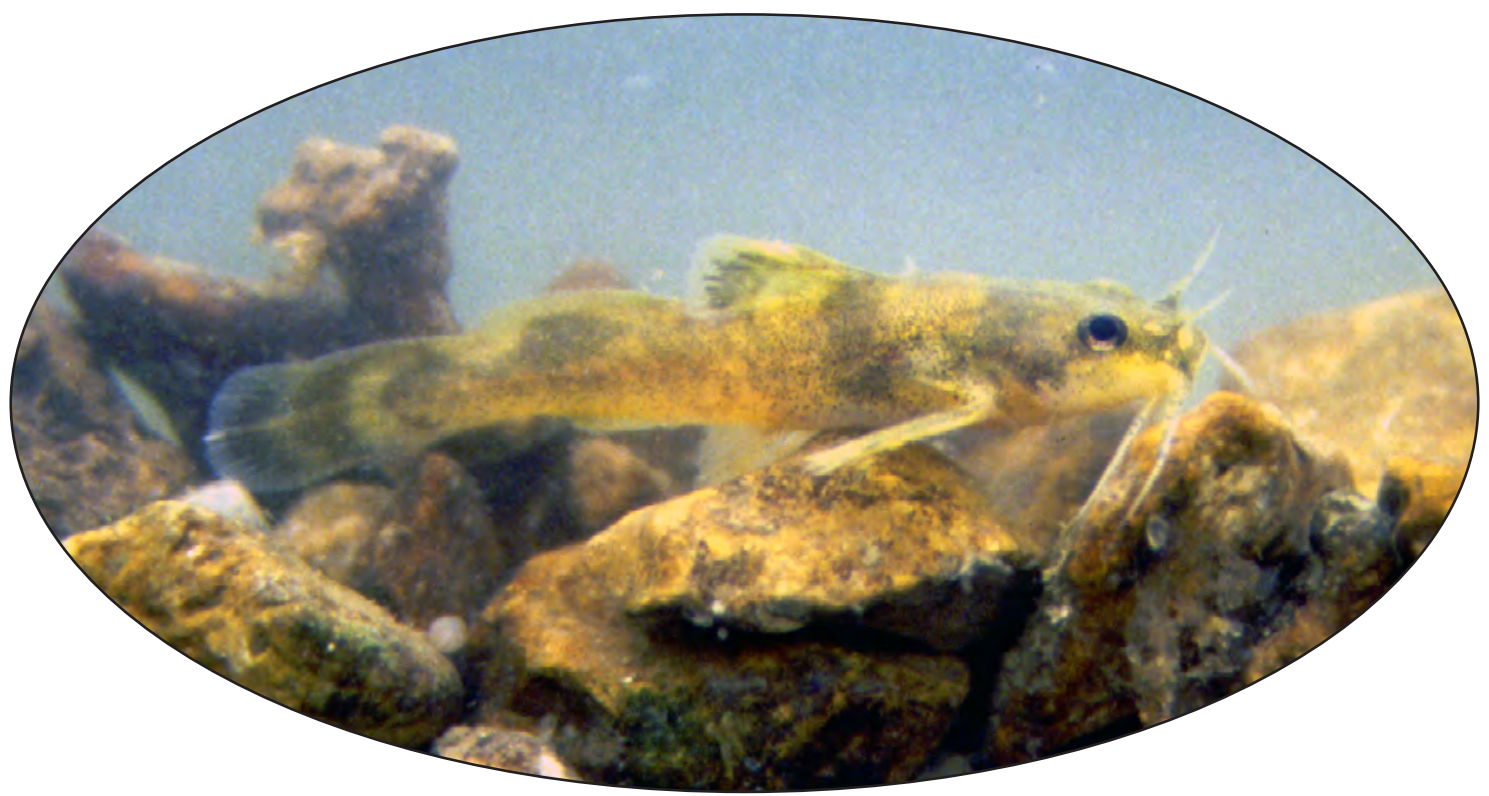

Open-File Report 2010-1109 
Cover photograph. Neosho madtom (Noturus placidus). Photograph by Janice L. Bryan, U.S. Geological Survey. 


\section{Neosho Madtom and Other Ictalurid Populations in Relation to Hydrologic Characteristics of an Impounded Midwestern Warmwater Stream: Update}

By Janice L. Bryan, Mark L. Wildhaber, William B. Leeds, and Rima Dey

Prepared in cooperation with the U.S. Fish and Wildlife Service

Open-File Report 2010-1109 


\title{
U.S. Department of the Interior \\ KEN SALAZAR, Secretary \\ U.S. Geological Survey \\ Marcia K. McNutt, Director
}

\section{U.S. Geological Survey, Reston, Virginia: 2010}

\author{
For more information on the USGS — the Federal source for science about the Earth, its natural and living resources, \\ natural hazards, and the environment, visit http://www.usgs.gov or call 1-888-ASK-USGS \\ For an overview of USGS information products, including maps, imagery, and publications, \\ visit http://www.usgs.gov/pubprod \\ To order this and other USGS information products, visit http://store.usgs.gov
}

\begin{abstract}
Any use of trade, product, or firm names is for descriptive purposes only and does not imply endorsement by the U.S. Government.

Although this report is in the public domain, permission must be secured from the individual copyright owners to reproduce any copyrighted materials contained within this report.
\end{abstract}

Suggested citation:

Bryan, J.B., Wildhaber, M.L., Leeds, W.B., and Dey, Rima, 2010, Neosho madtom and other ictalurid populations in relation to hydrologic characteristics of an impounded midwestern warmwater stream-update: U.S. Geological Survey Open-File Report 2010-1109, 20 p. with app. 


\section{Contents}

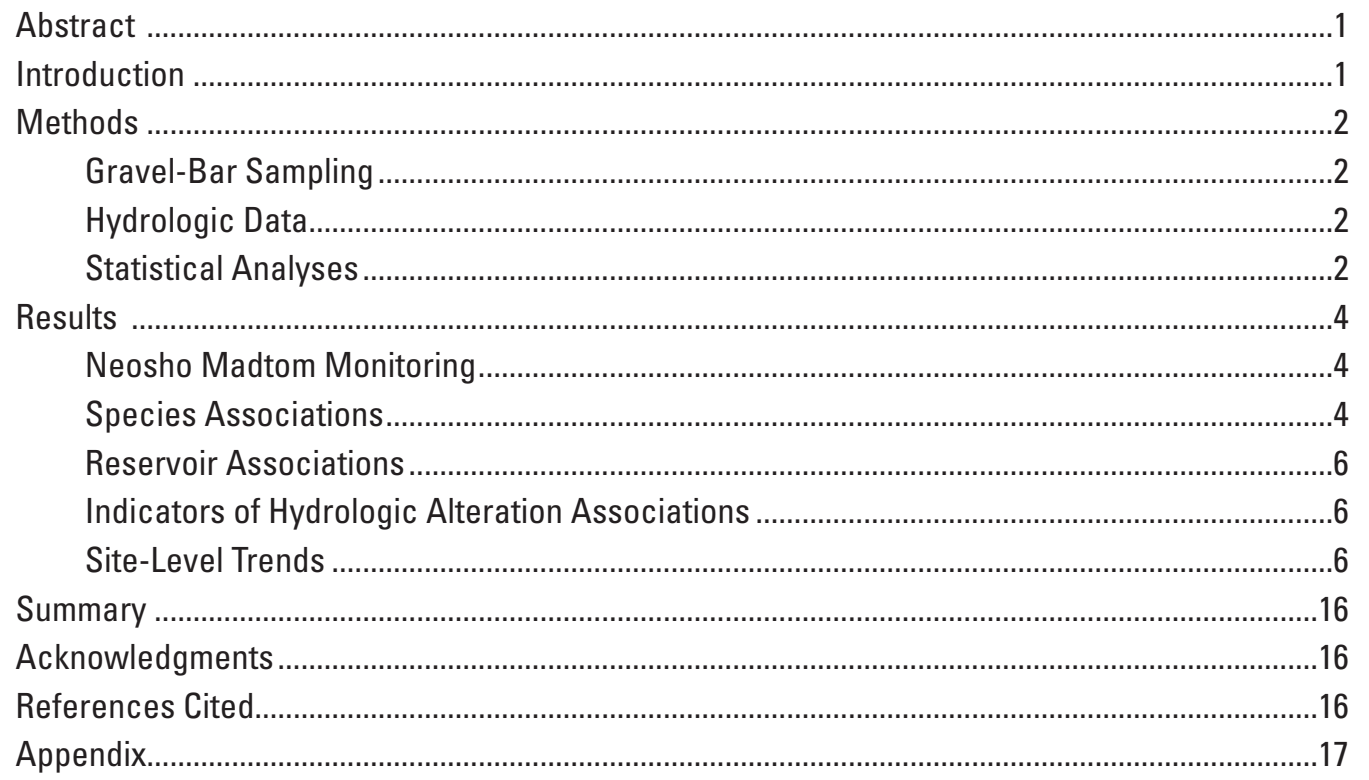

\section{Figures}

1. Map showing the position of the Neosho madtom sampling locations and U.S. Geological Survey streamgages used in this study. These locations are on the Neosho, Cottonwood, and Spring Rivers in Kansas, Missouri, and Oklahoma ...........3

2. Graph showing the pattern of the Indicators of Hydrologic Alteration parameters and Neosho madtom density at the U.S. Geological Survey streamgages on the Cottonwood/Neosho Rivers. The streamgages are ordered downstream, left to right; the dashed line indicates the location of the John Redmond Reservoir; and the letter ' $M$ ' indicates streamgage-level Neosho madtom density. 


\section{Tables}

1. Summary of the yearly mean value for Neosho madtom density per 36 square meters at each location and respective hydrologic streamgage used in this study ........5

2. Statistics about the relations between Neosho madtoms, other ictalurids, depth, and velocity. Statistics include: number of locations, mean, standard deviation, Pearson's correlation coefficients and their respective P-values

3. Two-way analysis of variance using Type III sums of squares and the associated $\mathrm{p}$-value, and $\mathrm{F}$ statistics for evaluating the variation in fish density and habitat variables due to year, position of the location relative to the John Redmond Reservoir, and their interaction

4. Coefficients and P-values from the two-way analysis of covariance of Neosho madtom densities and Indicators of Hydrologic Alteration parameters, summarized by river, preceding water year (water year prior to when the fish were sampled), and current water year (same year the fish were sampled). Neosho madtom densities were grouped and averaged for each year by the nearest U.S. Geological Survey streamgage

5. Slope of regression analysis and the P-value of the Durbin-Watson T-statistic for each site.

6. Site-level Pearson's correlation coefficients of Neosho madtom densities with the Indicators of Hydrologic Alteration parameters for the Cottonwood, Emporia, and Humbolt sites. Pearson's correlation coefficients and P-values are summarized for each site

7. Site-level Pearson's correlation coefficients of Neosho madtom densities with the Indicators of Hydrologic Alteration parameters for the National Wildlife Area, Oswego, and Oklahoma sites. Pearson's correlation coefficients and P-values are summarized for each site

8. Site-level Pearson's correlation coefficients of Neosho madtom densities with the Indicators of Hydrologic Alteration parameters for the Spring River sites.

Pearson's correlation coefficients and P-values are summarized for each site

\section{Appendix Tables}

1. Yearly median Indicators of Hydrologic Alteration parameters averaged over the years for the Cottonwood/Neosho River sites

2. Yearly median Indicators of Hydrologic Alteration parameters averaged over the years for the Spring River sites 


\section{Conversion Factors}

\begin{tabular}{|c|c|c|}
\hline Multiply & By & To obtain \\
\hline \multicolumn{3}{|c|}{ Length } \\
\hline inch (in.) & 2.54 & centimeter $(\mathrm{cm})$ \\
\hline centimeter $(\mathrm{cm})$ & 0.3937 & inch (in.) \\
\hline inch (in.) & 25.4 & millimeter $(\mathrm{mm})$ \\
\hline millimeter $(\mathrm{mm})$ & 0.03937 & inch (in.) \\
\hline foot $(\mathrm{ft})$ & 0.3048 & meter $(\mathrm{m})$ \\
\hline meter $(\mathrm{m})$ & 3.281 & foot $(\mathrm{ft})$ \\
\hline mile (mi) & 1.609 & kilometer $(\mathrm{km})$ \\
\hline kilometer $(\mathrm{km})$ & 0.6214 & mile (mi) \\
\hline \multicolumn{3}{|c|}{ Area } \\
\hline square inch $\left(\right.$ inch $\left.^{2}\right)$ & 645.16 & square millimeter $\left(\mathrm{mm}^{2}\right)$ \\
\hline square millimeter $\left(\mathrm{mm}^{2}\right)$ & 0.00155 & square inch $\left(\right.$ inch $\left.^{2}\right)$ \\
\hline square meter $\left(\mathrm{m}^{2}\right)$ & 10.76 & square foot $\left(\mathrm{ft}^{2}\right)$ \\
\hline square foot $\left(\mathrm{ft}^{2}\right)$ & 0.09290 & square meter $\left(\mathrm{m}^{2}\right)$ \\
\hline \multicolumn{3}{|c|}{ Volume } \\
\hline cubic meter $\left(\mathrm{m}^{3}\right)$ & 35.31 & cubic foot $\left(\mathrm{ft}^{3}\right)$ \\
\hline cubic foot $\left(\mathrm{ft}^{3}\right)$ & 0.02832 & cubic meter $\left(\mathrm{m}^{3}\right)$ \\
\hline \multicolumn{3}{|c|}{ Flow rate } \\
\hline cubic meter per second $\left(\mathrm{m}^{3} / \mathrm{s}\right)$ & 35.31 & cubic foot per second $\left(\mathrm{ft}^{3} / \mathrm{s}\right)$ \\
\hline cubic foot per second $\left(\mathrm{ft}^{3} / \mathrm{s}\right)$ & 0.02832 & cubic meter per second $\left(\mathrm{m}^{3} / \mathrm{s}\right)$ \\
\hline centimeter per second $(\mathrm{cm} / \mathrm{s})$ & 0.3937 & inch per second (in/s) \\
\hline inch per second (in/s) & 25.4 & centimeter per second $(\mathrm{cm} / \mathrm{s})$ \\
\hline meter per second $(\mathrm{m} / \mathrm{s})$ & 3.281 & foot per second $(\mathrm{ft} / \mathrm{s})$ \\
\hline foot per second $(\mathrm{ft} / \mathrm{s})$ & 0.3048 & meter per second $(\mathrm{m} / \mathrm{s})$ \\
\hline
\end{tabular}

Water year is the 12-month period from 0 ctober 1 through September 30 . The water year is designated by the calendar year in which the water year ends and that includes 9 of the 12 months. 



\title{
Neosho Madtom and Other Ictalurid Populations in Relation to Hydrologic Characteristics of an Impounded Midwestern Warmwater Stream: Update
}

\author{
By Janice L. Bryan' ${ }^{1}$, Mark L. Wildhaber ${ }^{1}$, William B. Leeds ${ }^{2}$, and Rima Dey²
}

\section{Abstract}

The Neosho madtom, Noturus placidus, is a small (less than 75 millimeters in total length) ictalurid that is native to the main stems of the Neosho and Cottonwood Rivers in Kansas and Oklahoma and the Spring River in Kansas and Missouri. The Neosho madtom was federally listed as threatened by the U.S. Fish and Wildlife Service in May 1990. The U.S. Fish and Wildlife Service has been monitoring Neosho madtoms since 1991, and questioned whether or not Neosho madtom densities were affected by other catfish species, reservoirs, and hydrologic characteristics. Using the first 8 years of U.S. Fish and Wildlife Service monitoring data, Wildhaber and others (2000) analyzed whether or not Neosho madtom densities were related to these environmental characteristics. The goal of this report is to update these results with data from 1999 to 2008. The trends of Neosho madtom densities in respect to John Redmond Reservoir and other catfish species remains consistent with the previous report. In both the Neosho and Spring Rivers, Neosho madtoms had a significant positive association with all catfish species. Of those species tested, only in the population of Neosho madtoms were significantly different in density above verses below the John Redmond Reservoir after accounting for the yearly variation. The average density of Neosho madtoms at the streamgage immediately below the reservoir had the second lowest density compared to the other streamgages. The positive associations with Neosho madtoms that remained consistent from the previous report included the 1-, 3-, and 7-day minima discharges and the annual minimum discharge from the previous water year (water year prior to when the fish were sampled) and the 1-, 3-, 7-, and 30-day minima discharges from the current water year (same water year fish were sampled).

\footnotetext{
${ }^{1}$ U.S. Geological Survey.

${ }^{2}$ University of Missouri-Columbia.
}

\section{Introduction}

The Neosho madtom, Noturus placidus, (Taylor, 1969) is a small [less than 75 millimeters $(\mathrm{mm})$ in total length] ictalurid that is native to the main stems of the Neosho and Cottonwood Rivers in Kansas and Oklahoma and the Spring River in Kansas and Missouri (Luttrell and others, 1992; Cross and Collins, 1995; Wilkinson and others, 1996). The Neosho madtom was federally listed as threatened by the U.S. Fish and Wildlife Service (USFWS) in May 1990 (U.S. Fish and Wildlife Service, 1991). This species occupies parts of riffles with mean current velocity of 79 centimeters per second $(\mathrm{cm} / \mathrm{s})$, mean depth of 0.23 meter $(\mathrm{m})$, and unconsolidated pieces of pebble and gravel substrate, 2 to 64 millimeters (mm) in diameter (Moss, 1983). Neosho madtoms feed at night on larval insects found among the gravel (Cross and Collins, 1995). Based on samples collected throughout the year (day and night), the greatest numbers of Neosho madtoms occur in riffles during daylight hours in late summer and early fall after young-of-year are believed to have been recruited to the population (Moss, 1983; Luttrell and others, 1992; Fuselier and Edds, 1994). Previous research indicates that Neosho madtoms have a life cycle that is annual in nature, with recruitment of young-of-year into adult collection gear about the time the adults begin to disappear from collections (Fuselier and Edds, 1994).

The USFWS has been monitoring Neosho madtoms since 1991. Using the first 8 years of USFWS monitoring data, Wildhaber and others (2000) analyzed whether or not Neosho madtom densities were affected by other catfish species, reservoirs, and hydrologic characteristics. The USFWS requested an update of this report including the years 1999 through 2008. In addition to the USFWS monitoring data, permission was given to use additional data from the Spring River collected by the Missouri Department of Conservation (MDC) as part of that agencies' conservation efforts for Neosho madtoms (Doug Novinger, Missouri Department of Conservation, oral commun., 2009). 


\section{Methods}

The following methods are relevant to the data collected by USFWS for this analysis, and specifics are described in Wildhaber and others (2000). Data from the Missouri section of the Spring River after 1995 were collected by MDC using slightly different methods (Doug Novinger, unpub. data, 2007-2008). The main difference between these two datasets are specified in the sections below; however, the methods were similar enough at the gravel-bar level that combining data is appropriate. Because of sampling, chemical, and geographic differences between the Spring River and the Cottonwood/ Neosho Rivers, we performed separate analyses for these two systems. A brief summary of relevant methods follows.

\section{Gravel-Bar Sampling}

From 1991 to 2008, gravel bars (shoreline collections of pebbles up to $38 \mathrm{~mm}$ in diameter that extend out into the river and were believed to provide suitable habitat for Neosho madtoms) were sampled by USFWS for monitoring Neosho madtom populations (U.S. Fish and Wildlife Service, 1991). Generally, the same gravel bars, or those in the same river reaches, were sampled each year. Sampling at all locations occurred during daylight hours between August and October, after Neosho madtom young-of-year recruitment was expected to have occurred.

Before sampling, three to five transects perpendicular to the river channel were equally spaced to span the length of the gravel bar. In most instances, five stations were spaced equally along each transect with a minimum distance of $2 \mathrm{~m}$ between adjacent stations. The MDC randomly selected five transects within six equal-length longitudinal sections of the gravel bar perpendicular to the river channel. Five stations along each transect were selected by MDC using a random distance offset (transect length multiplied by one-fifth, multiplied by random proportion). For all sampling, fewer than five stations were established if the river channel was less than $10 \mathrm{~m}$ wide or if a station occurred at a depth too great to seine (less than $1.25 \mathrm{~m}$ ). Transects on a gravel bar were sampled from downstream to upstream. On each transect, stations were sampled from nearest to most distant from the streambank.

At each location, sampling proceeded in the following order to minimize the impacts of samples on each other: fish were collected, then water depth and water velocity measurements were made. The counts of all benthic fishes were recorded, and the fish were released. This study analyzes only captured ictalurid species that included black bullhead, Ameiurus melas; blue catfish, Ictalurus furcatus; brindled madtom, N. miurus; channel catfish, I. punctatus; flathead catfish, Pylodictis olivaris; freckled madtom, N. nocturnus; Neosho madtom; slender madtom, $N$. exilis; stonecat, $N$. flavus; and Tadpole madtom, N. gyrinus. Fishes were collected from a 4.5-square meter $\left(\mathrm{m}^{2}\right)$ area by disturbing the substrate by kicking, starting $3 \mathrm{~m}$ upstream from a stationary seine (3.0-square millimeter $\left(\mathrm{mm}^{2}\right) \mathrm{mesh}$ ) and proceeding downstream to the seine. The MDC collected fish from a $3.6 \mathrm{~m}^{2}$ area by disturbing the substrate, starting $2 \mathrm{~m}$ upstream from a stationary seine $\left(3.2 \mathrm{~mm}^{2} \mathrm{mesh}\right)$. For all sampling, water depth and water velocity at 60 percent of water depth was measured.

\section{Hydrologic Data}

We obtained U.S Geological Survey (USGS) hydrologic data in the form of daily mean flows from streamgages on the Neosho, Cottonwood, and Spring Rivers. We used hydrologic data from the streamgages recorded during 1990 to 2008 at Americus, Plymouth, Burlington, Iola, and Parsons, Kans., Commerce, Okla., and Waco, Mo., to test relations among Indicators of Hydrologic Alteration (IHA) parameters and Neosho madtom densities (fig. 1).

For the sampling location downstream of the confluence of the Neosho and Cottonwood Rivers and upstream from John Redmond Reservoir (fig. 1), we added hydrologic data together from two streamgages, one near Americus, Kans. on the Neosho River and one near Plymouth, Kans. on the Cottonwood River. The streamgage near Americus is 38.6 kilometers $(\mathrm{km})$ upstream from the mouth of the Cottonwood River. The streamgage near Plymouth is $63.1 \mathrm{~km}$ upstream from the confluence with the Neosho River. The Americus and Plymouth streamgages are the nearest ones upstream from John Redmond Reservoir. There are no streamgages between the confluence of the Neosho and Cottonwood Rivers and the reservoir. Because the hydrologic data used consisted of daily means, the data from the Americus and Plymouth streamgages could be considered synchronized; therefore, as a conservative estimate, we summed the hydrologic data from these two streamgages to represent the Neosho River hydrograph above the reservoir and below the confluence streamgage the Neosho and Cottonwood Rivers.

\section{Statistical Analyses}

We analyzed the fish population and physical data to assess differences between locations above and below John Redmond Reservoir, as well as differences across years. Arithmetic means were calculated for depth and velocity. We calculated location densities for four categories of fish: Neosho madtoms, channel catfish, stonecats, and all nonNeosho madtom ictalurids combined. The last grouping was established because some species of ictalurids were not collected often enough to allow separate species-level analyses. We calculated fish densities by dividing the total number of Neosho madtoms, all other catfishes combined, channel catfish, and stonecats collected at a given location by the total area sampled by kickseining to arrive at fish density per square meter. Because differences were examined at the gravel-bar scale, we scaled the fish density up to that of the smallest bar sampled, which was $36 \mathrm{~m}^{2}$; consequently, all fish densities are reported from an area of $36 \mathrm{~m}^{2}$. 


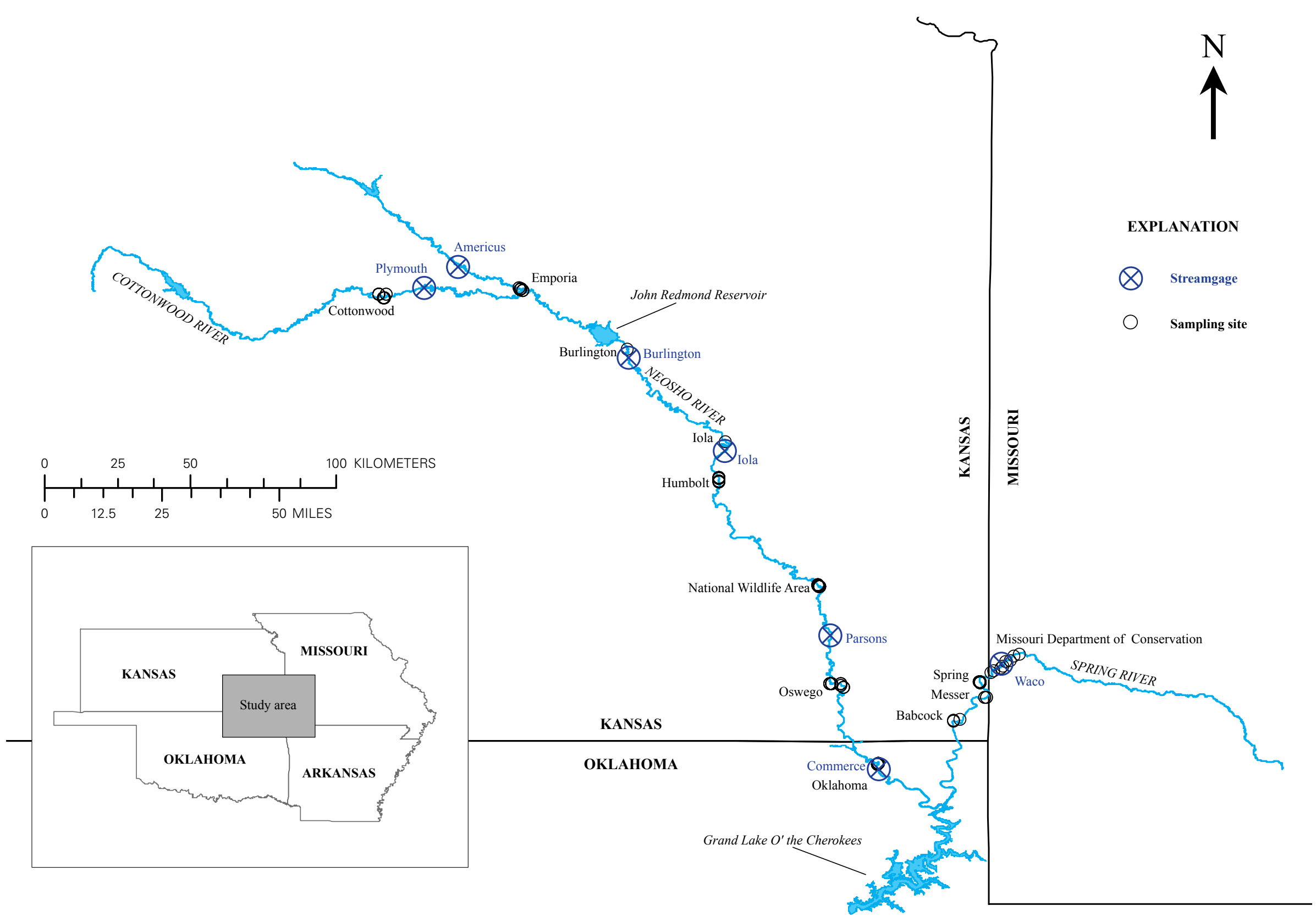

Figure 1. The position of the Neosho madtom sampling locations and U.S. Geological Survey streamgages used in this study. These locations are on the Neosho, Cottonwood, and Spring Rivers in Kansas, Missouri, and Oklahoma. 
Mean daily flow data were summarized before analysis using IHA parameter estimates produced with the Indicators of Hydrologic Alteration software, version 7.0.0 (Nature Conservancy, 1996-2006). All IHA parameters were calculated using nonparametric statistics; that is, by assuming the discharge data had a non-normal distribution. IHA nonparametric statistics use the median value except for the 1- to 90-day minima and maxima. We used nonparametric statistics instead of parametric statistics as in Wildhaber and others (2000), because of the non-normal distribution of the hydrologic data. As is standard in the IHA software, the four parameters relating to frequency and duration of high and low pulses were calculated relative to all years (1991-2008).

Because the primary interest in this analysis is whether the John Redmond Reservoir affects catfishes in the Neosho and Cottonwood Rivers, we conducted separate two-way Analyses of Variance (ANOVA) with year and position above or below the reservoir as factors. The ANOVAs were performed on values per sampling location, for fish densities, depth, and velocity. The ability to normalize variables through transformations and equal sample sizes allowed the use of the power and robustness of ANOVA to test for fish densities, depth, and velocity differences among hydrologic parameter mean values relative to the position above or below the reservoir despite the differences in variance that may have existed (Milliken and Johnson, 1984).

Pearson's correlation coefficients were used to assess relations between the densities of Neosho madtoms, all other catfishes combined, channel catfish, stonecats, depth, and velocity. A regression analysis was used to examine the slope of the relation between location-level Neosho madtom density and sampling year.

Separate two-way Analyses of Covariance (ANCOVA) were performed on Neosho madtom densities for each IHA parameter to assess the relations between densities and IHA parameters after adjusting for effects attributable to year and position relative to the reservoir. The above-the-reservoir versus below-the-reservoir association in the ANCOVA models accounted for relations that were the result of upstream to downstream patterns deriving from potentially important factors such as stream size and the presence of the reservoir. The goal of the ANCOVAs was to determine if Neosho madtom population trends were related to IHA parameters independent of the two main factors within the design of the study (that is, year and presence of the reservoir). For the ANCOVAs, we conducted separate analyses using the current (same water year the fish were sampled) and preceding water year (water year prior to when the fish were sampled) from the streamgage closest to the gravel bar sampled. Because there were fewer streamgages than there were gravel bars sampled, we grouped the gravel bars, referred to as locations, by nearest streamgage (that is, Plymouth, Americus, Plymouth and Americus combined, Burlington, Iola, Parsons, Commerce, or Waco; fig. 1). Using this grouped structure, the Neosho madtom location densities in each year were averaged by streamgage structure before any ANCOVAs were conducted. These averaged values are referred to as streamgage-level averages. Because there is only one streamgage on the Spring River, the analysis of that streamgage-level was simplified to a simple linear regression of IHA verses year. The associations between streamgagelevel Neosho madtom density averages and IHA parameters were examined using Pearson correlation coefficients.

Because of the annual nature of the life cycle of the Neosho madtom, it is possible to compare density relations with IHA parameters in the current and preceding water years to assess whether or not population trends were the result of survival to reproductive age (or reproductive success) and/ or survival of young-of-year to recruitment to the population, respectively. To confirm this assumption, we tested whether the yearly density of Neosho madtoms were independent by calculating the Durbin-Watson statistic for any ANOVA that did not have year as a discrete factor, and the Hoeffding dependence coefficient for all Pearson correlation coefficient analyses. These statistics were only calculated for tests where the number of observations was greater than five.

All statistical tests were conducted using SAS software version 9.1.3 (SAS, 2004). Means were checked for normality and tested for homogeneity of variance by using a Levene's test. Most non-normal variables were $\log _{10}+1$ transformed. All relations for which $P \leq 0.05$ were considered significant.

\section{Results}

\section{Neosho Madtom Monitoring}

During the 16 years of data collection, most of the monitoring locations have remained in similar areas (fig. 1). The number of locations sampled above and below John Redmond Reservoir were similar but varied somewhat owing to high-water conditions, shifting gravel bars, and budget and time constraints (table 1). In 1993, sampling was not possible because of extreme flooding. For this analysis, 195 locations were used (Cottonwood River, 20; Neosho River, 93; Spring River, 34; Spring River by MDC, 48). An additional 9 locations were sampled using non-standard procedures and they were not used in this analysis (table 1, Neosho River, 8; Spring River, 1). Two locations did not have depth and velocity data recorded, consequently 193 locations were used in the habitat analyses.

\section{Species Associations}

In the Cottonwood and Neosho Rivers, Neosho madtoms had a significant, positive association with all catfish species (table 2). The only difference between Neosho madtom density in the Cottonwood/Neosho and Spring Rivers was that Neosho madtom density had a significant positive association with velocity in the Cottonwood/Neosho Rivers and not in the Spring River. The Hoeffding coefficient, which measures independence, ranged from -0.01 to 0.05 . This range is not close 
Table 1. Summary of the yearly mean value for Neosho madtom density per 36 square meters at each location and respective hydrologic streamgage used in this study.

[Locations are ordered downstream, top to bottom. --, location was not sampled; a, standard protocol was not used and data were not included in analysis; NWA, National Wildlife Area; MDC, Missouri Department of Conservation]

\begin{tabular}{|c|c|c|c|c|c|c|c|c|c|c|c|c|c|c|c|c|c|c|c|c|c|c|}
\hline \multirow{2}{*}{ River } & \multirow{2}{*}{ Site } & \multirow{2}{*}{ Location } & \multirow{2}{*}{ Streamgage } & \multicolumn{18}{|c|}{ Year } & \multirow{2}{*}{$\begin{array}{l}\text { Mean } \\
\text { value }\end{array}$} \\
\hline & & & & 1991 & 1992 & 1993 & 1994 & 1995 & 1996 & 1997 & 1998 & 1999 & 2000 & 2001 & 2002 & 2003 & 2004 & 2005 & 2006 & 2007 & 2008 & \\
\hline \multicolumn{23}{|c|}{ Upstream from John Redmond Reservoir } \\
\hline \multirow[t]{3}{*}{ Cottonwood } & Cottonwood & 1 & Plymouth & 9.7 & 8.6 & -- & -- & -- & -- & -- & -- & -- & -- & -- & -- & -- & -- & -- & -- & -- & -- & 9.2 \\
\hline & & 2 & Plymouth & 8.8 & 10.7 & -- & 1.4 & -- & 1.3 & 4.0 & 6.3 & 3.1 & 0.7 & -- & -- & -- & 1.4 & -- & -- & -- & -- & 4.2 \\
\hline & & 3 & Plymouth & 19.3 & 25.9 & -- & 21.3 & -- & 1.3 & 7.0 & 13.0 & 16.7 & 7.0 & -- & -- & -- & 3.1 & -- & -- & -- & -- & 12.7 \\
\hline \multirow[t]{4}{*}{ Neosho } & Emporia & 1 & Americus & 0 & 2.4 & -- & -- & -- & -- & -- & -- & -- & -- & -- & -- & -- & -- & -- & -- & -- & -- & 1.2 \\
\hline & & 2 & Americus & 4.6 & 14.0 & -- & 24.9 & 7.5 & 5.3 & 0 & 2.5 & 0 & -- & -- & -- & -- & -- & -- & -- & -- & -- & 7.4 \\
\hline & & 3 & Americus & 6.4 & .3 & -- & -- & -- & -- & -- & -- & -- & -- & -- & -- & -- & -- & -- & -- & -- & -- & 3.4 \\
\hline & & 4 & Plymouth/Americus & -- & -- & -- & 72.7 & 19.8 & 2.9 & 20.9 & 45.7 & -- & -- & -- & -- & -- & -- & -- & -- & -- & -- & 32.4 \\
\hline \multicolumn{23}{|c|}{ Downstream from John Redmond Reservoir } \\
\hline \multirow{14}{*}{ Neosho } & Burlington & 1 & Burlington & -- & -- & -- & 0.6 & -- & -- & -- & -- & -- & -- & -- & -- & -- & -- & -- & -- & -- & -- & 0.6 \\
\hline & Iola & 1 & Iola & -- & -- & -- & -- & -- & -- & -- & -- & -- & -- & -- & -- & -- & -- & -- & -- & $\mathrm{a}$ & 7.4 & 7.4 \\
\hline & Humbolt & 1 & Iola & 4.9 & 40.0 & -- & -- & -- & -- & -- & -- & -- & -- & -- & -- & -- & -- & -- & -- & -- & -- & 22.4 \\
\hline & & 2 & Iola & 2.9 & 29.1 & -- & 4.5 & -- & -- & -- & -- & -- & 0.3 & -- & -- & -- & 1.6 & 2.2 & -- & -- & -- & 6.8 \\
\hline & & 3 & Iola & 5.9 & 38.0 & -- & 3.7 & -- & -- & -- & 21.1 & 39.7 & 5.1 & -- & -- & 1.0 & 6.4 & 1.3 & -- & $\mathrm{a}$ & 22.0 & 14.4 \\
\hline & NWA & 1 & Parsons & -- & -- & -- & 4.3 & 14.9 & -- & 4.2 & 5.8 & 16.4 & 5.8 & 3.8 & -- & 0 & 4.2 & 1.0 & 0 & -- & 11.2 & 6.0 \\
\hline & & 2 & Parsons & -- & -- & -- & 0 & 1.0 & -- & .3 & 4.2 & 1.3 & 0 & -- & -- & 0 & 1.3 & .3 & -- & -- & -- & .9 \\
\hline & Oswego & 1 & Parsons & 2.2 & 2.7 & -- & 1.3 & 1.6 & -- & -- & -- & -- & -- & -- & -- & -- & -- & -- & -- & $\mathrm{a}$ & -- & 1.9 \\
\hline & & 2 & Parsons & 0 & .5 & -- & -- & -- & -- & -- & -- & -- & -- & -- & -- & -- & -- & -- & -- & -- & -- & .3 \\
\hline & & 3 & Parsons & 0 & -- & -- & .6 & 1.0 & -- & .3 & .6 & 0 & 0 & 0 & 1.0 & 0 & .3 & 0 & $\mathrm{a}$ & $\mathrm{a}$ & -- & .3 \\
\hline & & 4 & Parsons & -- & -- & -- & -- & -- & -- & .3 & 1.7 & 1.5 & -- & -- & -- & -- & -- & -- & -- & -- & -- & 1.2 \\
\hline & Oklahoma & 1 & Commerce & -- & -- & -- & -- & -- & -- & 0 & -- & -- & -- & -- & -- & -- & -- & -- & -- & -- & -- & 0 \\
\hline & & 2 & Commerce & -- & -- & -- & -- & -- & -- & .4 & 1.3 & 1.0 & .3 & 0 & $\mathrm{a}$ & -- & -- & -- & -- & -- & -- & .6 \\
\hline & & 3 & Commerce & -- & -- & -- & -- & -- & -- & -- & 1.0 & 5.1 & 1.3 & 0 & 0 & 0 & 1.9 & 0 & $\mathrm{a}$ & $\mathrm{a}$ & -- & 1.2 \\
\hline \multirow{16}{*}{ Spring } & MDC & 20 & Waco & -- & -- & -- & 1.1 & -- & -- & -- & -- & -- & -- & -- & -- & .7 & .7 & 1.0 & 0 & 0.3 & 3.0 & 1.0 \\
\hline & & 30 & Waco & -- & -- & -- & 4.8 & -- & -- & -- & -- & -- & -- & -- & -- & 1.3 & 0 & 0 & 0 & 0 & .7 & 1.0 \\
\hline & & 40 & Waco & -- & -- & -- & -- & -- & -- & -- & -- & -- & -- & -- & -- & 0 & 0 & 0 & 0 & 0 & .3 & .1 \\
\hline & & 50 & Waco & -- & -- & -- & -- & -- & -- & -- & -- & -- & -- & -- & -- & 2.0 & 1.7 & 0 & 0 & 0 & 1.3 & .8 \\
\hline & & 60 & Waco & -- & -- & -- & -- & -- & -- & -- & -- & -- & -- & -- & -- & 1.3 & 1.0 & 1.7 & .7 & 0 & 3.0 & 1.3 \\
\hline & & 70 & Waco & -- & -- & -- & -- & -- & -- & -- & -- & -- & -- & -- & -- & 1.0 & 1.7 & 0 & .7 & 0 & 1.0 & .7 \\
\hline & & 80 & Waco & -- & -- & -- & -- & -- & -- & -- & -- & -- & -- & -- & -- & 0 & 0 & 0 & 0 & 0 & 0 & 0 \\
\hline & & 90 & Waco & -- & -- & -- & -- & 0 & -- & -- & -- & -- & -- & -- & -- & 0 & 0 & .3 & 0 & 0 & .3 & .1 \\
\hline & Spring & 1 & Waco & -- & -- & -- & .5 & 5.3 & -- & 0 & 0 & 1.7 & 0 & 0 & 0 & 0 & .4 & 0 & 0 & -- & -- & .7 \\
\hline & & 2 & Waco & -- & -- & -- & .8 & -- & -- & -- & -- & -- & -- & -- & -- & -- & -- & -- & -- & -- & -- & .8 \\
\hline & & 3 & Waco & -- & -- & -- & 0 & -- & -- & 0 & 0 & 0 & 0 & 0 & .6 & 0 & .3 & .3 & 0 & -- & -- & .1 \\
\hline & Messer & 1 & Waco & -- & -- & -- & -- & 1.0 & -- & -- & -- & -- & -- & -- & -- & -- & -- & -- & -- & -- & -- & 1.0 \\
\hline & & 2 & Waco & -- & -- & -- & 0 & -- & -- & -- & -- & -- & -- & -- & -- & -- & -- & 0 & $\mathrm{a}$ & -- & -- & 0 \\
\hline & Babcock & 1 & Waco & -- & -- & -- & -- & -- & -- & -- & -- & -- & -- & -- & -- & -- & -- & 0 & -- & -- & -- & 0 \\
\hline & & 2 & Waco & -- & -- & -- & -- & -- & -- & -- & -- & -- & -- & -- & -- & -- & -- & 0 & 0 & -- & -- & 0 \\
\hline & & 3 & Waco & -- & -- & -- & 0 & -- & -- & -- & -- & -- & -- & -- & -- & -- & -- & -- & -- & -- & -- & 0 \\
\hline
\end{tabular}


Table 2. Statistics about the relations between Neosho madtoms, other ictalurids, depth, and velocity. Statistics include: number of locations, mean, standard deviation, Pearson's correlation coefficients and their respective P-values.

[m, meter; $\mathrm{m} / \mathrm{sec}$, meter per second; $\mathrm{n}$, number of locations; na, not applicable; $<$, less than ]

\begin{tabular}{|c|c|c|c|c|c|c|}
\hline & $\begin{array}{l}\text { Neosho } \\
\text { madtoms }\end{array}$ & $\begin{array}{c}\text { Catfishes other } \\
\text { than Neosho } \\
\text { madtoms }\end{array}$ & $\begin{array}{l}\text { Channel } \\
\text { catfishes }\end{array}$ & Stonecats & $\begin{array}{c}\text { Depth } \\
\text { (m) }\end{array}$ & $\begin{array}{l}\text { Velocity } \\
\text { (m/sec) }\end{array}$ \\
\hline \multicolumn{7}{|c|}{ Cottonwood/Neosho Rivers } \\
\hline $\mathrm{n}$ & 113 & 113 & 113 & 113 & 111 & 111 \\
\hline Mean & 2.7796 & 9.8713 & 7.8767 & 1.0376 & 1.5676 & 1.0926 \\
\hline Pearson correlation coefficient & na & .4116 & .3754 & .3079 & -.0559 & .2288 \\
\hline Pearson coefficient $\mathrm{p}$-value & na & ${ }^{\mathrm{a}}<.0001$ & $\mathrm{a}<.0001$ & a .0009 & .5602 & ${ }^{\mathrm{a}} .0157$ \\
\hline Hoeffding coefficient & na & .05 & .03 & .03 & $<-.01$ & .02 \\
\hline \multicolumn{7}{|c|}{ Spring River } \\
\hline $\bar{n}$ & 82 & 82 & 82 & 82 & 82 & 82 \\
\hline Mean & .3436 & 3.8207 & .9153 & .4535 & 1.1569 & 1.5417 \\
\hline Pearson correlation coefficient & na & .3990 & .2415 & .2727 & -.0246 & .0198 \\
\hline Pearson coefficient $p$-value & na & a. 0002 & a .0288 & ${ }^{\mathrm{a}} .0132$ & .8267 & .8600 \\
\hline Hoeffding coefficient & na & .03 & $<-.01$ & $<-.01$ & -.01 & -.01 \\
\hline
\end{tabular}

${ }^{\text {a }}$ Indicates significance at the 0.05 level.

to 1 (the statistic can range from -0.05 to 1 ); consequently, the data are considered independent and the correlations valid (SAS, 2004).

\section{Reservoir Associations}

After 1998, only seven locations were sampled in 3 years upstream from John Redmond Reservoir; consequently, there were only 3 additional years in this analysis since the previous report (Wildhaber and others, 2000). Testing for reservoir associations limited the data to only those years where locations above and below the reservoir were sampled $(n=88)$. The position of the location above or below the reservoir explained a significant amount of the variation only in the Neosho madtom density after accounting for the yearly variation (table 3). None of the variation in either habitat variable was significantly explained by year or position relative to the reservoir.

\section{Indicators of Hydrologic Alteration Associations}

After averaging up to the streamgage level, there were 71 observations available with which to examine the association between hydrology and Neosho madtom density (Cottonwood/Neosho River, 57; Spring River, 14). The streamgagelevel densities immediately below the reservoir were the second lowest compared to the other densities (fig. 2). The locations near the Commerce streamgage had the lowest average density of Neosho madtoms and are the farthest downstream in the Neosho River (fig 1).
After removing the variation due to year and reservoir associations, the density of Neosho madtoms in the Cottonwood/Neosho Rivers was positively associated with the average June and October discharge, annual minimum, and 1-, 3-, and 7-day minima from the preceding water year (water year prior to when the fish were sampled; table 4). During the current water year (same water year the fish were sampled), the density of Neosho madtoms was positively associated with low-pulse count, 1-day maximum, and 1-, 3-, 7-, and 30-day minima. The range of the Durbin-Watson statistics for these 62 tests was 2.43 to 2.93, with a mean of 2.67. This would indicate independence of the observations for each test because they are close to 2 (range of statistic is 0 to 4 ; SAS, 2004).

After removing the variation due to year and reservoir associations, the density of Neosho madtoms in the Spring River was positively associated with the previous water year's average May and September discharge, and 7-day maximum (table 4). During the current water year, the density of Neosho madtoms was positively associated with average October and May discharge and rise rate. The range of the Durbin-Watson statistics for these 62 tests was 1.52 to 2.32 , with a mean of 1.90. This would indicate independence of the observations for each test because they are close to 2 (range of statistic is 0 to 4 ; SAS, 2004).

\section{Site-Level Trends}

The most years sampled at one location was 12, which occurred at three different locations (table 1). Neosho 
Table 3. Two-way analysis of variance using Type III sums of squares and the associated p-value, and $F$ statistics for evaluating the variation in fish density and habitat variables due to year, position of the location relative to the John Redmond Reservoir, and their interaction.

[Back-transformed mean values for the two locations also are summarized by species group and habitat variable. $\mathrm{m}^{2}$, square meter; $\mathrm{m}$, meter; $\mathrm{m} / \mathrm{sec}$, meter per second; F, F-distribution]

\begin{tabular}{|c|c|c|c|c|c|c|c|}
\hline \multirow[b]{2}{*}{ Variable } & & \multicolumn{4}{|c|}{ Density (fish per $36 \mathrm{~m}^{2}$ ) } & \multirow[b]{2}{*}{$\begin{array}{l}\text { Depth } \\
\text { (m) }\end{array}$} & \multirow[b]{2}{*}{$\begin{array}{l}\text { Velocity } \\
\text { (m/sec) }\end{array}$} \\
\hline & & $\begin{array}{l}\text { Neosho } \\
\text { madtoms }\end{array}$ & $\begin{array}{l}\text { Catfishes other } \\
\text { than Neosho } \\
\text { madtoms }\end{array}$ & $\begin{array}{l}\text { Channel } \\
\text { catfishes }\end{array}$ & Stonecats & & \\
\hline \multicolumn{8}{|c|}{ Type III sums of squares P-value (F) } \\
\hline Year & a8 & $\begin{array}{l}0.1342 \\
(1.62)\end{array}$ & $\begin{array}{l}{ }^{\mathrm{b}} 0.0113 \\
(2.72)\end{array}$ & $\begin{array}{l}{ }^{\mathrm{b}} 0.0057 \\
(3.02)\end{array}$ & $\begin{array}{l}0.3195 \\
(1.19)\end{array}$ & $\begin{array}{l}0.4778 \\
(.96)\end{array}$ & $\begin{array}{l}0.1696 \\
(1.51)\end{array}$ \\
\hline Location & ${ }^{\mathrm{a}} 1$ & $\begin{array}{l}\text { b.0043 } \\
(8.69)\end{array}$ & $\begin{array}{l}.2977 \\
(1.1)\end{array}$ & $\begin{array}{l}.2495 \\
(1.35)\end{array}$ & $\begin{array}{l}.4324 \\
(.62)\end{array}$ & $\begin{array}{l}.1878 \\
(1.77)\end{array}$ & $\begin{array}{l}.9026 \\
(.02)\end{array}$ \\
\hline Year/location interaction & a8 & $\begin{array}{l}.2492 \\
(1.32)\end{array}$ & $\begin{array}{l}{ }^{\mathrm{b}} .0200 \\
(2.48)\end{array}$ & $\begin{array}{l}{ }^{\mathrm{b}} .0176 \\
(2.53)\end{array}$ & $\begin{array}{l}.4108 \\
(1.05)\end{array}$ & $\begin{array}{l}.4281 \\
(1.02)\end{array}$ & $\begin{array}{l}.9330 \\
(.37)\end{array}$ \\
\hline \multicolumn{8}{|c|}{ Means } \\
\hline $\begin{array}{l}\text { Cottonwood and Neosho } \\
\text { River sites above John } \\
\text { Redmond Reservoir }\end{array}$ & c33 & 6.39 & 13.97 & 10.95 & 1.61 & 0.38 & 0.33 \\
\hline $\begin{array}{l}\text { Neosho River sites below } \\
\text { John Redmond Reservoir }\end{array}$ & c55 & 2.30 & 9.12 & 7.06 & 1.05 & .42 & .32 \\
\hline
\end{tabular}

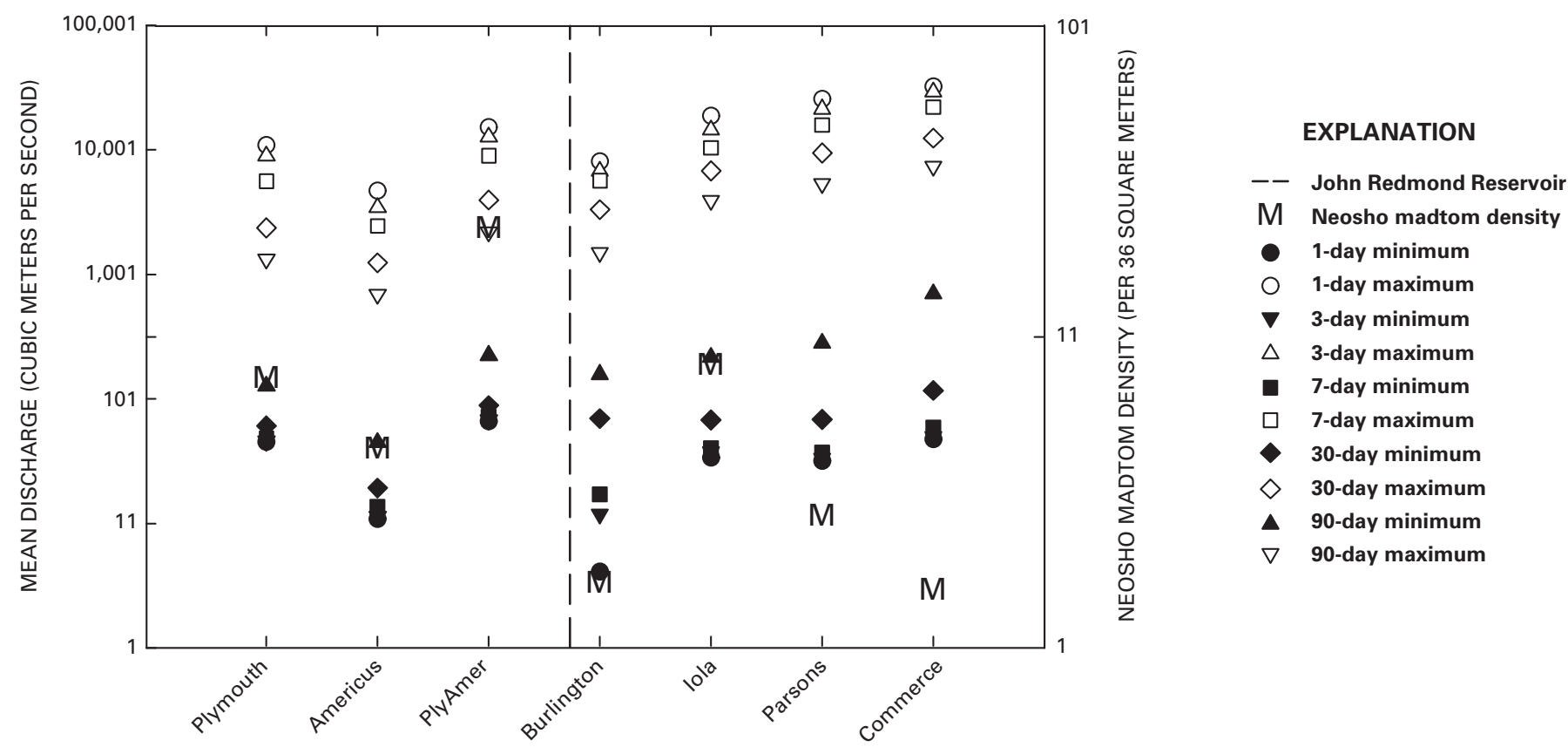

STREAMGAGE

Figure 2. The pattern of the Indicators of Hydrologic Alteration parameters and Neosho madtom density at the U.S. Geological Survey streamgages on the Cottonwood/Neosho Rivers. The streamgages are ordered downstream, left to right; the dashed line indicates the location of the John Redmond Reservoir; and the letter ' $M$ ' indicates streamgage-level Neosho madtom density. 
Table 4. Coefficients and P-values from the two-way analysis of covariance of Neosho madtom densities and Indicators of Hydrologic Alteration parameters, summarized by river, preceding water year (water year prior to when the fish were sampled), and current water year (same year the fish were sampled). Neosho madtom densities were grouped and averaged for each year by the nearest U.S. Geological Survey streamgage.

[The transformation in parentheses was performed on the indicated variables. $\mathrm{n}$, number of observations; $\mathrm{m}^{3} / \mathrm{sec}$, cubic meter per second; $\log _{10}$, logarithm with base $10 ;<$, less than]

\begin{tabular}{|c|c|c|c|c|c|c|c|c|}
\hline & \multicolumn{4}{|c|}{ Cottonwood/Neosho Rivers ( $=57$ ) } & \multicolumn{4}{|c|}{ Spring River ( $n=14$ ) } \\
\hline & \multicolumn{2}{|c|}{$\begin{array}{l}\text { Coefficient from } \\
\text { preceding water year } \\
\text { (P-value) }\end{array}$} & \multicolumn{2}{|c|}{$\begin{array}{l}\text { Coefficient from } \\
\text { current water year } \\
\text { (P-value) }\end{array}$} & \multicolumn{2}{|c|}{$\begin{array}{l}\text { Coefficient from } \\
\text { preceding water year } \\
\text { (P-value) }\end{array}$} & \multicolumn{2}{|c|}{$\begin{array}{l}\text { Coefficient from } \\
\text { current water year } \\
\text { (P-value) }\end{array}$} \\
\hline \multicolumn{9}{|c|}{ Monthly discharge $\left(\mathrm{m}^{3} / \mathrm{sec}\right)$} \\
\hline October $\left(\log _{10}+1\right)$ & 0.5577 & ${ }^{\mathrm{a}}(0.0198)$ & 0.6013 & ${ }^{\mathrm{b}}(0.0511)$ & 0.0736 & $(0.5048)$ & 0.1974 & $\mathrm{a}(0.0494)$ \\
\hline November $\left(\log _{10}+1\right)$ & .4671 & $\mathrm{~b}(.0534)$ & .1131 & $(.7109)$ & .0238 & $(.7514)$ & .0965 & $(.1991)$ \\
\hline December $\left(\log _{10}+1\right)$ & .3485 & $(.1849)$ & .1817 & $(.5037)$ & .0790 & $(.4031)$ & .1052 & $(.3236)$ \\
\hline January $\left(\log _{10}+1\right)$ & .4062 & $(.1111)$ & .3281 & $(.2597)$ & .0828 & $(.3706)$ & .0991 & $(.3267)$ \\
\hline Feburary $\left(\log _{10}+1\right)$ & .3656 & $(.1373)$ & .3262 & $(.2531)$ & .0945 & $(.3042)$ & .0772 & $(.4139)$ \\
\hline $\operatorname{March}\left(\log _{10}+1\right)$ & .3250 & $(.2105)$ & .3116 & $(.2691)$ & .1106 & $(.2329)$ & .0512 & $(.6375)$ \\
\hline April $\left(\log _{10}+1\right)$ & .6418 & ${ }^{\mathrm{b}}(.0688)$ & .4934 & $(.1250)$ & .1967 & $\mathrm{a}(.0395)$ & .1884 & $\mathrm{a}(.0500)$ \\
\hline May $\left(\log _{10}+1\right)$ & .3424 & $(.3040)$ & .5135 & $(.1139)$ & .2062 & $(.1289)$ & .2830 & $\mathrm{a}(.0021)$ \\
\hline June $\left(\log _{10}+1\right)$ & .5124 & ${ }^{\mathrm{a}}(.0411)$ & .3424 & $(.1589)$ & .1870 & $(.1557)$ & .1823 & $\mathrm{~b}(.0838)$ \\
\hline July $\left(\log _{10}+1\right)$ & .3849 & $(.1042)$ & .4524 & $\mathrm{~b}(.0840)$ & .1016 & $(.3624)$ & .1143 & $(.3335)$ \\
\hline August $\left(\log _{10}+1\right)$ & .3697 & $(.1566)$ & .3773 & $(.1531)$ & .1982 & $(.1556)$ & .1453 & $(.3175)$ \\
\hline September $\left(\log _{10}+1\right)$ & .2769 & $(.2694)$ & .3367 & $(.1552)$ & .2874 & $\mathrm{a}(.0129)$ & .1205 & $(.4052)$ \\
\hline \multicolumn{9}{|c|}{ Magnitude and duration of annual extremes $\left(\mathrm{m}^{3} / \mathrm{sec}\right)$} \\
\hline 1-day minimum $\left(\log _{10}+1\right)$ & 0.4680 & $\mathrm{a}(0.0226)$ & 0.6223 & $\mathrm{a}(0.0031)$ & 0.1489 & $(0.3677)$ & 0.2263 & $(0.2015)$ \\
\hline 1-day maximum $\left(\log _{10}+1\right)$ & .2436 & $(.4517)$ & 6170 & ${ }^{\mathrm{a}}(.0379)$ & .1720 & $\mathrm{~b}(.0660)$ & .1093 & $(.3111)$ \\
\hline 3-day minimum $\left(\log _{10}+1\right)$ & .6003 & $\mathrm{a}(.0089)$ & .7527 & $\mathrm{a}(.0011)$ & .1521 & $(.3704)$ & .2392 & $(.1868)$ \\
\hline 3-day maximum $\left(\log _{10}+1\right)$ & .0818 & $(.7794)$ & .4721 & $\mathrm{~b}(.0923)$ & .1695 & $\mathrm{~b}(.0670)$ & .1185 & $(.2615)$ \\
\hline 7 -day minimum $\left(\log _{10}+1\right)$ & .5997 & ${ }^{\mathrm{a}}(.0118)$ & .7819 & ${ }^{\mathrm{a}}(.0011)$ & .1630 & $(.3507)$ & .2570 & $(.1675)$ \\
\hline 7 -day maximum $\left(\log _{10}+1\right)$ & -.0004 & $(.9988)$ & .3975 & $(.1647)$ & .1954 & $\mathrm{a}(.0469)$ & .1204 & $(.2925)$ \\
\hline 30-day minimum $\left(\log _{10}+1\right)$ & .4015 & $\mathrm{~b}(.0992)$ & .6930 & ${ }^{\mathrm{a}}(.0097)$ & .1689 & $(.3542)$ & .2949 & $(.1307)$ \\
\hline 30-day maximum $\left(\log _{10}+1\right)$ & .2467 & $(.4856)$ & .5732 & $\mathrm{~b}(.0922)$ & .2120 & ${ }^{\mathrm{b}}(.0645)$ & .1653 & $(.1537)$ \\
\hline 90-day minimum $\left(\log _{10}+1\right)$ & .2178 & $(.3796)$ & .1858 & $(.4925)$ & .0611 & $(.5661)$ & .1626 & $(.1732)$ \\
\hline 90-day maximum $\left(\log _{10}+1\right)$ & .3063 & $(.3969)$ & .5472 & $(.1127)$ & .2266 & $\mathrm{~b}(.0799)$ & .2084 & ${ }^{b}(.0803)$ \\
\hline \multicolumn{9}{|c|}{ Timing of annual extremes (day of the year) } \\
\hline Annual minimum & 0.0063 & $\mathrm{a}(0.0082)$ & $<0.0001$ & $(0.9929)$ & -0.0012 & $(0.4965)$ & 0.0005 & $(0.7659)$ \\
\hline Annual maximum & .0028 & $(.1322)$ & .0011 & $(.5412)$ & .0001 & $(.8209)$ & .0006 & $(.3741)$ \\
\hline \multicolumn{9}{|c|}{ Rate and frequency of change in conditions } \\
\hline Fall rate $\left(\mathrm{m}^{3} / \mathrm{sec}, \log _{10}+1\right)$ & 0.1916 & $(0.5946)$ & 0.3962 & $(0.2262)$ & 0.1668 & $(0.1228)$ & 0.1656 & $(0.2185)$ \\
\hline Rise rate $\left(\mathrm{m}^{3} / \mathrm{sec}, \log _{10}+1\right)$ & -.0171 & $(.9486)$ & .1116 & $(.6738)$ & .1010 & $(.1576)$ & .1396 & $\mathrm{a}(.0453)$ \\
\hline $\begin{array}{l}\text { Number of reversals between rising } \\
\text { and falling discharges }\end{array}$ & .0076 & $(.1895)$ & -.0018 & $(.8101)$ & -.0057 & $(.2948)$ & -.0027 & $(.6241)$ \\
\hline
\end{tabular}

Frequency and duration of low and high pulses

\begin{tabular}{lrlllllll}
\hline Low-pulse length in days $\left(\log _{10}+1\right)$ & -0.4880 & $\mathrm{~b}(0.0548)$ & -0.3639 & $(0.1185)$ & -0.1161 & $(0.1642)$ & 0.0943 & $(0.3379)$ \\
Low-pulse count $\left(\log _{10}+1\right)$ & -.5490 & $(.2904)$ & -.9032 & $\mathrm{a}(.0344)$ & -.2126 & $(.3268)$ & -.3745 & $(.1835)$ \\
High-pulse length in days & .0144 & $(.6447)$ & -.0366 & $(.3477)$ & .0371 & $\mathrm{~b}(.0599)$ & .0483 & $(.1655)$ \\
High-pulse count & -.0408 & $(.3156)$ & .0044 & $(.9074)$ & .0079 & $(.4762)$ & -.0028 & $(.8099)$ \\
\hline
\end{tabular}

${ }^{a}$ Indicates significance at the 0.05 level.

${ }^{\mathrm{b}}$ Indicates significance at the 0.10 level. 
madtoms never were detected in five locations on the Spring River. The greatest density of Neosho madtoms at one sampling location was 32.4 per $36 \mathrm{~m}^{2}$ from Emporia, Kans., location number 4 (table 1). Sampling locations in all three rivers indicated a decrease in Neosho madtom density from 1991 to 2006, with a slight increase in density occurring in 2008 (table 1). After averaging up to the site level, all sites had a negative slope over the years, although this trend was significant at only the Oswego site on the Neosho River (table 5). The range of the Durbin-Watson statistics for these nine tests was 1.89 to 2.72 . This would indicate independence of the observations for each test because they are close to 2 (range of statistic is 0 to 4 ; SAS, 2004).

After averaging the sampling locations up to the site level (mean IHA parameters are given in the appendix;
Cottonwood/Neosho Rivers, 66 total observations from 7 sites; Spring River, 23 total observations from 3 sites), there were many negative associations between average Neosho madtom densities and the IHA parameters, but only 30 of 620 comparisons were statistically significant (tables 6-8). The Hoeffding coefficient for the Cottonwood/Neosho River sites ranged from -0.16 to 0.40 , with a mean of -0.01 , and -0.18 to 0.18 with a mean of -0.04 , for the current and previous water years analysis, respectively. For the Spring River sites, it ranged from -0.13 to 0.33 , with a mean of -0.0003 and -0.20 to 0.39 , with a mean of -0.02 for the current and previous water years analysis, respectively. These ranges are not close to 1 (the statistic can range from -0.05 to 1 ), consequently the data are considered independent and the correlations valid (SAS, 2004).

Table 5. Slope of regression analysis and the P-value of the Durbin-Watson T-statistic for each site.

[--, not enough data to perform analysis; NWA, National Wildlife Area; MDC, Missouri Department of Conservation; *, due to no variation in density, no P-value can be calculated]

\begin{tabular}{llcccc}
\hline \multicolumn{1}{c}{ River } & \multicolumn{1}{c}{ Site } & $\begin{array}{c}\text { Total } \\
\text { years }\end{array}$ & Slope & P-value & $\begin{array}{c}\text { Durbin-Watson } \\
\text { T-statistic }\end{array}$ \\
\hline Cottonwood & Cottonwood & 9 & -0.0447 & ${ }^{\mathrm{a}} 0.0782$ & 1.90 \\
Neosho & Emporia & 8 & -.0972 & .1337 & 1.86 \\
& Emporia location 4 & 5 & -.0375 & .8455 & 1.60 \\
& Burlington & 1 & -- & -- & -- \\
& Iola & 1 & -- & -- & -- \\
& Humbolt & 10 & -.0182 & .5528 & 2.08 \\
& NWA & 12 & -.0222 & .3884 & 2.17 \\
\multirow{5}{*}{ Spring } & Oswego & 13 & -.0231 & ${ }^{\mathrm{b}} .0095$ & 2.69 \\
& Oklahoma & 9 & -.0232 & .4764 & 1.92 \\
& MDC & 8 & -.0124 & .4195 & 2.41 \\
& Spring & 12 & -.0281 & .1187 & 2.72 \\
& Messer & 3 & -.0121 & .7191 & 2.64 \\
& Babcock & 3 & 0 & $*$ & $*$ \\
\hline
\end{tabular}

a Indicates significance at the 0.10 level.

${ }^{\mathrm{b}}$ Indicates significance at the 0.05 level. 
Table 6. Site-level Pearson's correlation coefficients of Neosho madtom densities with the Indicators of Hydrologic Alteration parameters for the Cottonwood, Emporia, and Humbolt sites. Pearson's correlation coefficients and P-values are summarized for each site.

[The transformation in parentheses was performed on the indicated variables. $\mathrm{n}$, number of years; $\mathrm{m}^{3} / \mathrm{sec}$, cubic meter per second; $\log _{10}$, $\operatorname{logarithm}$ with base 10]

\begin{tabular}{|c|c|c|c|c|c|c|c|c|}
\hline \multirow[b]{2}{*}{ Parameter } & \multicolumn{4}{|c|}{ Cottonwood $(n=9)$} & \multicolumn{4}{|c|}{ Emporia ( $n=8)$} \\
\hline & \multicolumn{2}{|c|}{$\begin{array}{l}\text { Coefficient from } \\
\text { preceding water year } \\
\text { (P-value) }\end{array}$} & \multicolumn{2}{|c|}{$\begin{array}{l}\text { Coefficient from } \\
\text { current water year } \\
\text { (P-value) }\end{array}$} & \multicolumn{2}{|c|}{$\begin{array}{l}\text { Coefficient from } \\
\text { preceding water year } \\
\text { (P-value) }\end{array}$} & \multicolumn{2}{|c|}{$\begin{array}{l}\text { Coefficient from } \\
\text { current water year } \\
\text { (P-value) }\end{array}$} \\
\hline \multicolumn{9}{|c|}{ Monthly discharge $\left(\mathrm{m}^{3} / \mathrm{sec}\right)$} \\
\hline October $\left(\log _{10}+1\right)$ & 0.2353 & $(0.5422)$ & -0.2348 & $(0.543)$ & -0.0793 & $(0.8519)$ & -0.5727 & $(0.1379)$ \\
\hline November $\left(\log _{10}+1\right)$ & .2496 & $(.5172)$ & -.0959 & $(.8061)$ & .3211 & $(.4381)$ & -.7887 & ${ }^{\mathrm{a}}(.0200)$ \\
\hline December $\left(\log _{10}+1\right)$ & .2536 & $(.5103)$ & -.1786 & $(.6456)$ & .2191 & $(.6022)$ & -.5883 & $(.1250)$ \\
\hline January $\left(\log _{10}+1\right)$ & .3776 & $(.3164)$ & -.0429 & $(.9127)$ & .1906 & $(.6511)$ & -.4848 & $(.2234)$ \\
\hline Feburary $\left(\log _{10}+1\right)$ & .3492 & $(.3570)$ & -.0459 & $(.9066)$ & .2501 & $(.5503)$ & -.6619 & ${ }^{\mathrm{b}}(.0738)$ \\
\hline $\operatorname{March}\left(\log _{10}+1\right)$ & .2472 & $(.5213)$ & -.1649 & $(.6716)$ & .1858 & $(.6596)$ & -.4089 & $(.3145)$ \\
\hline April $\left(\log _{10}+1\right)$ & .0321 & $(.9346)$ & .1750 & $(.6524)$ & .5265 & $(.1801)$ & -.5470 & $(.1606)$ \\
\hline May $\left(\log _{10}+1\right)$ & -.4547 & $(.2188)$ & -.0653 & $(.8674)$ & .4316 & $(.2856)$ & -.1637 & $(.6985)$ \\
\hline June $\left(\log _{10}+1\right)$ & -.4371 & $(.2394)$ & -.3105 & $(.4162)$ & .3680 & $(.3698)$ & -.0555 & $(.8961)$ \\
\hline July $\left(\log _{10}+1\right)$ & .0249 & $(.9494)$ & -.0719 & $(.8541)$ & .3499 & $(.3956)$ & .0046 & $(.9913)$ \\
\hline August $\left(\log _{10}+1\right)$ & -.1594 & $(.6821)$ & -.3031 & $(.4279)$ & .2652 & $(.5256)$ & .0663 & $(.8761)$ \\
\hline September $\left(\log _{10}+1\right)$ & -.5758 & $(.1047)$ & -.5149 & $(.1560)$ & .3376 & $(.4135)$ & .1739 & $(.6805)$ \\
\hline \multicolumn{9}{|c|}{ Magnitude and duration of annual extremes $\left(\mathrm{m}^{3} / \mathrm{sec}\right)$} \\
\hline 1-day minimum $\left(\log _{10}+1\right)$ & 0.2467 & $(0.5222)$ & -0.4971 & $(0.1733)$ & 0.0998 & $(0.8141)$ & -0.4669 & $(0.2435)$ \\
\hline 1 -day maximum $\left(\log _{10}+1\right)$ & -.3410 & $(.3691)$ & -.0482 & $(.9021)$ & .0923 & $(.8279)$ & -.4967 & $(.2105)$ \\
\hline 3-day minimum $\left(\log _{10}+1\right)$ & .2159 & $(.5768)$ & -.4954 & $(.1750)$ & .1731 & $(.6819)$ & -.4613 & $(.2499)$ \\
\hline 3-day maximum $\left(\log _{10}+1\right)$ & -.3860 & $(.3048)$ & -.1207 & $(.7570)$ & .1257 & $(.7668)$ & -.4442 & $(.2701)$ \\
\hline 7-day minimum $\left(\log _{10}+1\right)$ & .2296 & $(.5524)$ & -.4822 & $(.1886)$ & .2650 & $(.5260)$ & -.6161 & $(.1038)$ \\
\hline 7-day maximum $\left(\log _{10}+1\right)$ & -.4400 & $(.2359)$ & -.0769 & $(.8442)$ & .1157 & $(.7850)$ & -.3173 & $(.4437)$ \\
\hline 30-day minimum $\left(\log _{10}+1\right)$ & .2650 & $(.4908)$ & -.4765 & $(.1947)$ & .0856 & $(.8403)$ & -.4457 & $(.2684)$ \\
\hline 30 -day maximum $\left(\log _{10}+1\right)$ & -.4557 & $(.2176)$ & -.0961 & $(.8058)$ & .2009 & $(.6333)$ & -.2721 & $(.5144)$ \\
\hline 90 -day minimum $\left(\log _{10}+1\right)$ & .3722 & $(.3239)$ & -.1887 & $(.6268)$ & .2744 & $(.5108)$ & -.5447 & $(.1627)$ \\
\hline 90-day maximum $\left(\log _{10}+1\right)$ & -.3232 & $(.3963)$ & -.0847 & $(.8285)$ & .2918 & $(.4831)$ & -.3544 & $(.3890)$ \\
\hline \multicolumn{9}{|c|}{ Timing of annual extremes (day of the year) } \\
\hline Annual minimum & -0.0246 & $(0.9499)$ & 0.5883 & $\mathrm{~b}(0.0956)$ & 0.5828 & $(0.1295)$ & -0.0546 & $(0.8979)$ \\
\hline Annual maximum & -.2884 & $(.4517)$ & .1618 & $(.6775)$ & .6478 & ${ }^{\mathrm{b}}(.0824)$ & -.3459 & $(.4013)$ \\
\hline \multicolumn{9}{|c|}{ Rate and frequency of change in conditions } \\
\hline Fall rate $\left(\mathrm{m}^{3} / \mathrm{sec}, \log _{10}+1\right)$ & 0.0178 & $(0.9637)$ & 0.1234 & $(0.7517)$ & 0.4554 & $(0.2568)$ & -0.7069 & $\mathrm{a}(0.0499)$ \\
\hline Rise rate $\left(\mathrm{m}^{3} / \mathrm{sec}, \log _{10}+1\right)$ & .1751 & $(.6523)$ & .0857 & $(.8264)$ & .3860 & $(.3450)$ & -.5901 & $(.1236)$ \\
\hline $\begin{array}{l}\text { Number of reversals between rising } \\
\text { and falling discharges }\end{array}$ & .0801 & $(.8377)$ & .1060 & $(.7861)$ & .3873 & $(.3431)$ & -.3679 & $(.3699)$ \\
\hline \multicolumn{9}{|c|}{ Frequency and duration of low and high pulses } \\
\hline Low-pulse length in days $\left(\log _{10}+1\right)$ & -0.3184 & $(0.4037)$ & 0.1909 & $(0.6227)$ & -0.1730 & $(0.6820)$ & 0.3674 & $(0.3706)$ \\
\hline Low-pulse count $\left(\log _{10}+1\right)$ & .0950 & $(.8078)$ & .1101 & $(.7780)$ & -.3980 & $(.3289)$ & .6639 & ${ }^{b}(.0726)$ \\
\hline High-pulse length in days & .3916 & $(.2972)$ & -.6976 & $\mathrm{a}(.0367)$ & .5016 & $(.2054)$ & -.5893 & $(.1242)$ \\
\hline High-pulse count & -.1113 & $(.7756)$ & .0524 & $(.8935)$ & -.4574 & $(.2545)$ & -.3997 & $(.3266)$ \\
\hline
\end{tabular}


Table 6. Site-level Pearson's correlation coefficients of Neosho madtom densities with the Indicators of Hydrologic Alteration parameters for the Cottonwood, Emporia, and Humbolt sites. Pearson's correlation coefficients and P-values are summarized for each site.-Continued

[The transformation in parentheses was performed on the indicated variables. n, number of years; $\mathrm{m}^{3} / \mathrm{sec}$, cubic meter per second; $\log _{10}$, $\log _{\text {arithm }}$ with base 10]

\begin{tabular}{|c|c|c|c|c|c|c|c|c|}
\hline \multirow[b]{2}{*}{ Parameter } & \multicolumn{4}{|c|}{ Emporia location $4(n=5)$} & \multicolumn{4}{|c|}{ Humbolt $(n=10)$} \\
\hline & \multicolumn{2}{|c|}{$\begin{array}{l}\text { Coefficient from } \\
\text { preceding water year } \\
\text { (P-value) }\end{array}$} & \multicolumn{2}{|c|}{$\begin{array}{l}\text { Coefficient from } \\
\text { current water year } \\
\text { (P-value) }\end{array}$} & \multicolumn{2}{|c|}{$\begin{array}{c}\text { Coefficient from } \\
\text { preceding water year } \\
\text { (P-value) }\end{array}$} & \multicolumn{2}{|c|}{$\begin{array}{l}\text { Coefficient from } \\
\text { current water year } \\
\text { (P-value) }\end{array}$} \\
\hline \multicolumn{9}{|c|}{ Monthly discharge $\left(\mathrm{m}^{3} / \mathrm{sec}\right)$} \\
\hline October $\left(\log _{10}+1\right)$ & 0.7058 & $(0.1829)$ & 0.3477 & $(0.5664)$ & -0.1798 & $(0.6191)$ & 0.2982 & $(0.4026)$ \\
\hline November $\left(\log _{10}+1\right)$ & .9378 & $\mathrm{a}(.0185)$ & .2088 & $(.7361)$ & -.2212 & $(.5391)$ & .2892 & $(.4177)$ \\
\hline December $\left(\log _{10}+1\right)$ & .8946 & ${ }^{\mathrm{a}}(.0404)$ & .4316 & $(.4681)$ & .0121 & $(.9736)$ & .2784 & $(.4361)$ \\
\hline January $\left(\log _{10}+1\right)$ & .8689 & $\mathrm{~b}(.0559)$ & .4582 & $(.4377)$ & .1817 & $(.6154)$ & .2780 & $(.4368)$ \\
\hline Feburary $\left(\log _{10}+1\right)$ & .8183 & $\mathrm{~b}(.0904)$ & .4696 & $(.4249)$ & -.0899 & $(.8050)$ & .2930 & $(.4112)$ \\
\hline $\operatorname{March}\left(\log _{10}+1\right)$ & .3098 & $(.6120)$ & .4964 & $(.3949)$ & -.0795 & $(.8271)$ & .3267 & $(.3568)$ \\
\hline April $\left(\log _{10}+1\right)$ & .5188 & $(.3704)$ & .6958 & $(.1920)$ & .0821 & $(.8217)$ & .5407 & $(.1065)$ \\
\hline May $\left(\log _{10}+1\right)$ & -.1935 & $(.7552)$ & -.0197 & $(.9750)$ & -.2356 & $(.5123)$ & .1776 & $(.6235)$ \\
\hline June $\left(\log _{10}+1\right)$ & -.4232 & $(.4777)$ & -.3469 & $(.5673)$ & -.5139 & $(.1287)$ & .1103 & $(.7615)$ \\
\hline July $\left(\log _{10}+1\right)$ & .4250 & $(.4757)$ & .3713 & $(.5384)$ & -.0505 & $(.8898)$ & .5261 & $(.1183)$ \\
\hline August $\left(\log _{10}+1\right)$ & .3215 & $(.5978)$ & -.7844 & $(.1162)$ & .1130 & $(.7559)$ & .5627 & $\mathrm{~b}(.0904)$ \\
\hline September $\left(\log _{10}+1\right)$ & .2741 & $(.6554)$ & -.9307 & $\mathrm{a}(.0217)$ & -.0855 & $(.8144)$ & -.0972 & $(.7893)$ \\
\hline \multicolumn{9}{|c|}{ Magnitude and duration of annual extremes $\left(\mathrm{m}^{3} / \mathrm{sec}\right)$} \\
\hline 1-day minimum $\left(\log _{10}+1\right)$ & 0.8417 & $\mathrm{~b}(0.0738)$ & -0.1729 & $(0.7810)$ & 0.1526 & $(0.6738)$ & -0.0486 & $(0.8939)$ \\
\hline 1-day maximum $\left(\log _{10}+1\right)$ & .1922 & $(.7569)$ & -.5232 & $(.3656)$ & -.0788 & $(.8287)$ & .3663 & $(.2978)$ \\
\hline 3-day minimum $\left(\log _{10}+1\right)$ & .8419 & $\mathrm{~b}(.0736)$ & -.1743 & $(.7792)$ & .2369 & $(.5099)$ & -.0466 & $(.8982)$ \\
\hline 3-day maximum $\left(\log _{10}+1\right)$ & .1830 & $(.7683)$ & -.3819 & $(.5258)$ & -.1671 & $(.6446)$ & .2859 & $(.4233)$ \\
\hline 7-day minimum $\left(\log _{10}+1\right)$ & .8350 & $\mathrm{~b}(.0784)$ & -.1866 & $(.7637)$ & .3084 & $(.3859)$ & -.0386 & $(.9156)$ \\
\hline 7-day maximum $\left(\log _{10}+1\right)$ & .0219 & $(.9722)$ & -.2510 & $(.6838)$ & -.2738 & $(.4440)$ & .2087 & $(.5628)$ \\
\hline 30 -day minimum $\left(\log _{10}+1\right)$ & .8696 & $\mathrm{~b}(.0554)$ & -.1366 & $(.8266)$ & .1710 & $(.6367)$ & .1017 & $(.7798)$ \\
\hline 30-day maximum $\left(\log _{10}+1\right)$ & -.1447 & $(.8164)$ & -.2338 & $(.7050)$ & -.3376 & $(.3401)$ & .3584 & $(.3092)$ \\
\hline 90-day minimum $\left(\log _{10}+1\right)$ & .8551 & $\mathrm{~b}(.0648)$ & .4091 & $(.4941)$ & -.0225 & $(.9508)$ & .4067 & $(.2435)$ \\
\hline 90-day maximum $\left(\log _{10}+1\right)$ & .0086 & $(.9891)$ & -.1732 & $(.7806)$ & -.2959 & $(.4064)$ & .4675 & $(.1730)$ \\
\hline \multicolumn{9}{|c|}{ Timing of annual extremes (day of the year) } \\
\hline Annual minimum & -0.0077 & $(0.9901)$ & 0.2750 & $(0.6544)$ & -0.0072 & $(0.9841)$ & -0.3540 & $(0.3156)$ \\
\hline Annual maximum & -.2395 & $(.6980)$ & -.8643 & $\mathrm{~b}(.0588)$ & -.2258 & $(.5306)$ & .5425 & $(.1052)$ \\
\hline \multicolumn{9}{|c|}{ Rate and frequency of change in conditions } \\
\hline Fall rate $\left(\mathrm{m}^{3} / \mathrm{sec}, \log _{10}+1\right)$ & 0.5178 & $(0.3715)$ & 0.4174 & $(0.4844)$ & -0.0755 & $(0.8358)$ & 0.4759 & $(0.1644)$ \\
\hline Rise rate $\left(\mathrm{m}^{3} / \mathrm{sec}, \log _{10}+1\right)$ & .7153 & $(.1744)$ & .3512 & $(.5622)$ & -.1413 & $(.6970)$ & .4712 & $(.1692)$ \\
\hline $\begin{array}{l}\text { Number of reversals between rising } \\
\text { and falling discharges }\end{array}$ & -.0529 & $(.9326)$ & -.2115 & $(.7328)$ & -.2637 & $(.4616)$ & .2761 & $(.4401)$ \\
\hline \multicolumn{9}{|c|}{ Frequency and duration of low and high pulses } \\
\hline Low-pulse length in days $\left(\log _{10}+1\right)$ & -0.9123 & $\mathrm{a}(0.0308)$ & 0.0780 & $(0.9007)$ & -0.3148 & $(0.3756)$ & -0.1677 & $(0.6433)$ \\
\hline Low-pulse count $\left(\log _{10}+1\right)$ & -.3147 & $(.6060)$ & -.3843 & $(.5230)$ & -.3222 & $(.3638)$ & -.1831 & $(.6126)$ \\
\hline High-pulse length in days & .1458 & $(.8151)$ & -.7757 & $(.1231)$ & -.4318 & $(.2128)$ & .3477 & $(.3249)$ \\
\hline High-pulse count & .3295 & $(.5882)$ & .0893 & $(.8864)$ & .3451 & $(.3287)$ & .3064 & $(.3892)$ \\
\hline
\end{tabular}

a Indicates significance at the 0.05 level.

${ }^{\mathrm{b}}$ Indicates significance at the 0.10 level. 
Table 7. Site-level Pearson's correlation coefficients of Neosho madtom densities with the Indicators of Hydrologic Alteration parameters for the National Wildlife Area, Oswego, and Oklahoma sites. Pearson's correlation coefficients and P-values are summarized for each site.

[The transformation in parentheses was performed on the indicated variables. NWA, National Wildlife Area; $\mathrm{n}$, number of years; $\mathrm{m}^{3} / \mathrm{sec}$, cubic meter per second; $\log _{10}$, logarithm with base 10]

\begin{tabular}{|c|c|c|c|c|c|c|c|c|}
\hline \multirow[b]{2}{*}{ Parameter } & \multicolumn{4}{|c|}{ NWA ( $n=12)$} & \multicolumn{4}{|c|}{ Oswego (n= 13) } \\
\hline & \multicolumn{2}{|c|}{$\begin{array}{c}\text { Coefficient from } \\
\text { preceding water year } \\
\text { (P-value) }\end{array}$} & \multicolumn{2}{|c|}{$\begin{array}{l}\text { Coefficient from } \\
\text { current water year } \\
\text { (P-value) }\end{array}$} & \multicolumn{2}{|c|}{$\begin{array}{l}\text { Coefficient from } \\
\text { preceding water year } \\
\text { (P-value) }\end{array}$} & \multicolumn{2}{|c|}{$\begin{array}{l}\text { Coefficient from } \\
\text { current water year } \\
\text { (P-value) }\end{array}$} \\
\hline \multicolumn{9}{|c|}{ Monthly discharge $\left(\mathrm{m}^{3} / \mathrm{sec}\right)$} \\
\hline October $\left(\log _{10}+1\right)$ & 0.1669 & $(0.6041)$ & 0.3155 & $(0.3178)$ & -0.0961 & $(0.7549)$ & 0.1250 & $(0.6840)$ \\
\hline November $\left(\log _{10}+1\right)$ & -.1020 & $(.7524)$ & .2432 & $(.4463)$ & .0480 & $(.8764)$ & .1321 & $(.6671)$ \\
\hline December $\left(\log _{10}+1\right)$ & .1582 & $(.6234)$ & .3047 & $(.3356)$ & -.0285 & $(.9263)$ & .0822 & $(.7895)$ \\
\hline January $\left(\log _{10}+1\right)$ & -.0069 & $(.9829)$ & .4291 & $(.1640)$ & .1418 & $(.6440)$ & -.0171 & $(.9558)$ \\
\hline Feburary $\left(\log _{10}+1\right)$ & -.0899 & $(.7811)$ & .4128 & $(.1823)$ & .0414 & $(.8932)$ & -.2616 & $(.3880)$ \\
\hline $\operatorname{March}\left(\log _{10}+1\right)$ & .1410 & $(.6620)$ & .5464 & $\mathrm{a}(.0661)$ & -.0353 & $(.9089)$ & -.3256 & $(.2777)$ \\
\hline April $\left(\log _{10}+1\right)$ & .4502 & $(.1420)$ & .7013 & $\mathrm{~b}(.0110)$ & .1045 & $(.7341)$ & .0551 & $(.8580)$ \\
\hline May $\left(\log _{10}+1\right)$ & .0382 & $(.9063)$ & .1784 & $(.5791)$ & .0208 & $(.9461)$ & .2956 & $(.3268)$ \\
\hline June $\left(\log _{10}+1\right)$ & -.5364 & ${ }^{\mathrm{a}}(.0722)$ & .4670 & $(.1259)$ & -.3340 & $(.2646)$ & -.1697 & $(.5794)$ \\
\hline July $\left(\log _{10}+1\right)$ & -.0811 & $(.8022)$ & .5993 & $\mathrm{~b}(.0394)$ & -.1400 & $(.6482)$ & .2818 & $(.3509)$ \\
\hline August $\left(\log _{10}+1\right)$ & .1784 & $(.5791)$ & .7045 & $\mathrm{~b}(.0105)$ & .1903 & $(.5335)$ & .3711 & $(.2118)$ \\
\hline September $\left(\log _{10}+1\right)$ & -.1223 & $(.7049)$ & .0525 & $(.8714)$ & .0873 & $(.7768)$ & -.3763 & $(.2050)$ \\
\hline \multicolumn{9}{|c|}{ Magnitude and duration of annual extremes $\left(\mathrm{m}^{3} / \mathrm{sec}\right)$} \\
\hline 1-day minimum $\left(\log _{10}+1\right)$ & 0.0625 & $(0.8471)$ & 0.0694 & $(0.8303)$ & 0.1696 & $(0.5796)$ & -0.1705 & $(0.5777)$ \\
\hline 1-day maximum $\left(\log _{10}+1\right)$ & .3523 & $(.2614)$ & .5750 & $\mathrm{a}(.0505)$ & .0412 & $(.8936)$ & .4580 & $(.1155)$ \\
\hline 3-day minimum $\left(\log _{10}+1\right)$ & .0558 & $(.8632)$ & .0604 & $(.8520)$ & .1562 & $(.6104)$ & -.1598 & $(.6019)$ \\
\hline 3-day maximum $\left(\log _{10}+1\right)$ & .2307 & $(.4706)$ & .5093 & $\mathrm{a}(.0908)$ & .0225 & $(.9418)$ & .4192 & $(.1540)$ \\
\hline 7 -day minimum $\left(\log _{10}+1\right)$ & .0071 & $(.9824)$ & .0531 & $(.8699)$ & .1245 & $(.6852)$ & -.1564 & $(.6098)$ \\
\hline 7-day maximum $\left(\log _{10}+1\right)$ & .1894 & $(.5555)$ & .4432 & $(.1490)$ & -.0610 & $(.8430)$ & .4007 & $(.1748)$ \\
\hline 30-day minimum $\left(\log _{10}+1\right)$ & -.0256 & $(.9370)$ & -.0004 & $(.9990)$ & .2205 & $(.4692)$ & -.1321 & $(.6671)$ \\
\hline 30-day maximum $\left(\log _{10}+1\right)$ & .0775 & $(.8108)$ & .4682 & $(.1247)$ & -.1178 & $(.7015)$ & .3630 & $(.2229)$ \\
\hline 90 -day minimum $\left(\log _{10}+1\right)$ & -.0714 & $(.8256)$ & .3204 & $(.3099)$ & .0452 & $(.8833)$ & .1087 & $(.7237)$ \\
\hline 90-day maximum $\left(\log _{10}+1\right)$ & .0584 & $(.8568)$ & .6036 & $(.0377)$ & -.1325 & $(.6662)$ & .3653 & $(.2197)$ \\
\hline \multicolumn{9}{|c|}{ Timing of annual extremes (day of the year) } \\
\hline Annual minimum & -0.2186 & $(0.4950)$ & 0.2335 & $(0.4651)$ & 0.0207 & $(0.9465)$ & -0.0224 & $(0.9422)$ \\
\hline Annual maximum & .1661 & $(.6059)$ & .2874 & $(.3651)$ & -.2388 & $(.4321)$ & .2462 & $(.4174)$ \\
\hline \multicolumn{9}{|c|}{ Rate and frequency of change in conditions } \\
\hline Fall rate $\left(\mathrm{m}^{3} / \mathrm{sec}, \log _{10}+1\right)$ & 0.1156 & $(0.7206)$ & 0.6522 & $\mathrm{~b}(0.0215)$ & 0.0125 & $(0.9677)$ & 0.0131 & $(0.9661)$ \\
\hline Rise rate $\left(\mathrm{m}^{3} / \mathrm{sec}, \log _{10}+1\right)$ & .2018 & $(.5294)$ & .7677 & $\mathrm{~b}(.0035)$ & .1217 & $(.6921)$ & .2017 & $(.5088)$ \\
\hline $\begin{array}{l}\text { Number of reversals between rising } \\
\text { and falling discharges }\end{array}$ & .0811 & $(.8022)$ & .3559 & $(.2562)$ & .1913 & $(.5312)$ & -.1316 & $(.6681)$ \\
\hline \multicolumn{9}{|c|}{ Frequency and duration of low and high pulses } \\
\hline Low-pulse length in days $\left(\log _{10}+1\right)$ & 0.0092 & $(0.9774)$ & -0.3973 & $(0.2009)$ & 0.1219 & $(0.6916)$ & -0.0997 & $(0.7459)$ \\
\hline Low-pulse count $\left(\log _{10}+1\right)$ & -.2327 & $(.4668)$ & -.2954 & $(.3513)$ & -.5302 & $\mathrm{a}(.0623)$ & -.2243 & $(.4613)$ \\
\hline High-pulse length in days & -.4434 & $(.1488)$ & -.1131 & $(.7264)$ & -.2605 & $(.3901)$ & .2015 & $(.5092)$ \\
\hline High-pulse count & .1967 & $(.5401)$ & .5700 & $\mathrm{a}(.0530)$ & -.0236 & $(.9390)$ & -.2792 & $(.3556)$ \\
\hline
\end{tabular}


Table 7. Site-level Pearson's correlation coefficients of Neosho madtom densities with the Indicators of Hydrologic Alteration parameters for the National Wildlife Area, Oswego, and Oklahoma sites. Pearson's correlation coefficients and P-values are summarized for each site.-Continued

[The transformation in parentheses was performed on the indicated variables. NWA, National Wildlife Area; $\mathrm{n}$, number of years; $\mathrm{m}^{3} /$ sec, cubic meter per second; $\log _{10}$, logarithm with base 10$]$

\begin{tabular}{|c|c|c|c|c|}
\hline \multirow[b]{2}{*}{ Parameter } & \multicolumn{4}{|c|}{ Oklahoma $(n=9)$} \\
\hline & \multicolumn{2}{|c|}{$\begin{array}{c}\text { Coefficient from } \\
\text { preceding water year } \\
\text { (P-value) }\end{array}$} & \multicolumn{2}{|c|}{$\begin{array}{c}\text { Coefficient from } \\
\text { current water year } \\
\text { (P-value) }\end{array}$} \\
\hline \multicolumn{5}{|c|}{ Monthly discharge $\left(\mathrm{m}^{3} / \mathrm{sec}\right)$} \\
\hline October $\left(\log _{10}+1\right)$ & 0.3558 & $(0.3474)$ & 0.7573 & ${ }^{\mathrm{b}}(0.0181)$ \\
\hline November $\left(\log _{10}+1\right)$ & .3148 & $(.4093)$ & .3872 & $(.3032)$ \\
\hline December $\left(\log _{10}+1\right)$ & .3319 & $(.3830)$ & .7004 & ${ }^{\mathrm{b}}(.0356)$ \\
\hline January $\left(\log _{10}+1\right)$ & .4124 & $(.2700)$ & .5110 & $(.1598)$ \\
\hline Feburary $\left(\log _{10}+1\right)$ & -.0077 & $(.9843)$ & .3724 & $(.3236)$ \\
\hline $\operatorname{March}\left(\log _{10}+1\right)$ & .1679 & $(.6658)$ & .4406 & $(.2352)$ \\
\hline April $\left(\log _{10}+1\right)$ & .4593 & $(.2136)$ & .6214 & ${ }^{\mathrm{a}}(.0740)$ \\
\hline May $\left(\log _{10}+1\right)$ & .3435 & $(.3654)$ & .2111 & $(.5855)$ \\
\hline June $\left(\log _{10}+1\right)$ & -.7271 & ${ }^{\mathrm{b}}(.0264)$ & .1131 & $(.7721)$ \\
\hline July $\left(\log _{10}+1\right)$ & .0681 & $(.8618)$ & .4909 & $(.1796)$ \\
\hline August $\left(\log _{10}+1\right)$ & .1763 & $(.6501)$ & .2881 & $(.4522)$ \\
\hline September $\left(\log _{10}+1\right)$ & .6136 & ${ }^{\mathrm{a}}(.0788)$ & -.4338 & $(.2434)$ \\
\hline \multicolumn{5}{|c|}{ Magnitude and duration of annual extremes $\left(\mathrm{m}^{3} / \mathrm{sec}\right)$} \\
\hline 1-day minimum $\left(\log _{10}+1\right)$ & 0.4485 & $(0.2260)$ & 0.2484 & $(0.5192)$ \\
\hline 1-day maximum $\left(\log _{10}+1\right)$ & -.1401 & $(.7191)$ & .5372 & $(.1358)$ \\
\hline 3 -day minimum $\left(\log _{10}+1\right)$ & .4359 & $(.2408)$ & .2583 & $(.5023)$ \\
\hline 3 -day maximum $\left(\log _{10}+1\right)$ & -.1320 & $(.7349)$ & .4638 & $(.2086)$ \\
\hline 7 -day minimum $\left(\log _{10}+1\right)$ & .3800 & $(.3131)$ & .2510 & $(.5147)$ \\
\hline 7 -day maximum $\left(\log _{10}+1\right)$ & .0003 & $(.9993)$ & .5007 & $(.1697)$ \\
\hline 30-day minimum $\left(\log _{10}+1\right)$ & .4466 & $(.2281)$ & .2144 & $(.5795)$ \\
\hline 30-day maximum $\left(\log _{10}+1\right)$ & .0529 & $(.8925)$ & .4059 & $(.2784)$ \\
\hline 90 -day minimum $\left(\log _{10}+1\right)$ & .3080 & $(.4201)$ & .4958 & $(.1746)$ \\
\hline 90-day maximum $\left(\log _{10}+1\right)$ & .1555 & $(.6895)$ & .4974 & $(.1731)$ \\
\hline \multicolumn{5}{|c|}{ Timing of annual extremes (day of the year) } \\
\hline Annual minimum & -0.6112 & $\mathrm{a}(0.0803)$ & -0.1079 & $(0.7822)$ \\
\hline Annual maximum & .0268 & $(.9454)$ & .5692 & $(.1097)$ \\
\hline \multicolumn{5}{|c|}{ Rate and frequency of change in conditions } \\
\hline Fall rate $\left(\mathrm{m}^{3} / \mathrm{sec}, \log _{10}+1\right)$ & 0.2555 & $(0.5070)$ & 0.5565 & $(0.1196)$ \\
\hline Rise rate $\left(\mathrm{m}^{3} / \mathrm{sec}, \log _{10}+1\right)$ & .2907 & $(.4479)$ & .5837 & $\mathrm{a}(.0989)$ \\
\hline $\begin{array}{l}\text { Number of reversals between rising } \\
\text { and falling discharges }\end{array}$ & .6618 & ${ }^{\mathrm{a}}(.0522)$ & .2424 & $(.5298)$ \\
\hline \multicolumn{5}{|c|}{ Frequency and duration of low and high pulses } \\
\hline Low-pulse length in days $\left(\log _{10}+1\right)$ & 0.0786 & $(0.8407)$ & -0.3857 & $(0.3052)$ \\
\hline Low-pulse count $\left(\log _{10}+1\right)$ & -.8172 & ${ }^{\mathrm{b}}(.0072)$ & -.0424 & $(.9137)$ \\
\hline High-pulse length in days & .1237 & $(.7512)$ & .6024 & ${ }^{\mathrm{a}}(.0860)$ \\
\hline High-pulse count & .2312 & $(.5494)$ & .1350 & $(.7291)$ \\
\hline
\end{tabular}

${ }^{a}$ Indicates significance at the 0.10 level.

${ }^{\mathrm{b}}$ Indicates significance at the 0.05 level. 
Table 8. Site-level Pearson's correlation coefficients of Neosho madtom densities with the Indicators of Hydrologic Alteration parameters for the Spring River sites. Pearson's correlation coefficients and P-values are summarized for each site.

[The transformation in parentheses was performed on the indicated variables. MDC, Missouri Department of Conservation; $\mathrm{n}, \mathrm{number}$ of years; $\mathrm{m}^{3} /$ sec, cubic meter per second; $\log _{10}$, logarithm with base 10$]$

\begin{tabular}{|c|c|c|c|c|c|c|c|c|}
\hline \multirow[b]{2}{*}{ Parameter } & \multicolumn{4}{|c|}{$\operatorname{MDC}(n=8)$} & \multicolumn{4}{|c|}{ Spring ( $=12$ ) } \\
\hline & \multicolumn{2}{|c|}{$\begin{array}{l}\text { Coefficient from } \\
\text { preceding water year } \\
\text { (P-value) }\end{array}$} & \multicolumn{2}{|c|}{$\begin{array}{l}\text { Coefficient from } \\
\text { current water year } \\
\text { (P-value) }\end{array}$} & \multicolumn{2}{|c|}{$\begin{array}{l}\text { Coefficient from } \\
\text { preceding water year } \\
\text { (P-value) }\end{array}$} & \multicolumn{2}{|c|}{$\begin{array}{c}\text { Coefficient from } \\
\text { current water year } \\
\text { (P-value) }\end{array}$} \\
\hline \multicolumn{9}{|c|}{ Monthly discharge $\left(\mathrm{m}^{3} / \mathrm{sec}\right)$} \\
\hline October $\left(\log _{10}+1\right)$ & -0.0289 & $(0.9459)$ & 0.6696 & $\mathrm{a}(0.0693)$ & 0.4370 & $(0.1555)$ & 0.4297 & $(0.1632)$ \\
\hline November $\left(\log _{10}+1\right)$ & .1844 & $(.6620)$ & .1181 & $(.7807)$ & .1652 & $(.6079)$ & .4973 & ${ }^{\mathrm{b}}(.0100)$ \\
\hline December $\left(\log _{10}+1\right)$ & .2471 & $(.5552)$ & .3217 & $(.4371)$ & .2007 & $(.5317)$ & .3028 & $(.3388)$ \\
\hline January $\left(\log _{10}+1\right)$ & .3623 & $(.3778)$ & -.0448 & $(.9161)$ & .0799 & $(.8050)$ & .4492 & $(.1429)$ \\
\hline Feburary $\left(\log _{10}+1\right)$ & .3899 & $(.3397)$ & .1407 & $(.7396)$ & .0550 & $(.8651)$ & .2949 & $(.3521)$ \\
\hline $\operatorname{March}\left(\log _{10}+1\right)$ & .4363 & $(.2798)$ & .4432 & $(.2714)$ & .2176 & $(.4970)$ & .0798 & $(.8052)$ \\
\hline April $\left(\log _{10}+1\right)$ & .4284 & $(.2896)$ & .4303 & $(.2872)$ & .4218 & $(.1720)$ & .3920 & $(.2076)$ \\
\hline May $\left(\log _{10}+1\right)$ & .4520 & $(.2609)$ & -.0785 & $(.8535)$ & .1373 & $(.6706)$ & .8137 & ${ }^{b}(.0013)$ \\
\hline June $\left(\log _{10}+1\right)$ & .8240 & ${ }^{\mathrm{b}}(.0119)$ & -.0584 & $(.8907)$ & -.0087 & $(.9785)$ & .7390 & ${ }^{\mathrm{b}}(.0060)$ \\
\hline July $\left(\log _{10}+1\right)$ & .7483 & ${ }^{\mathrm{b}}(.0327)$ & -.0176 & $(.9671)$ & -.0860 & $(.7905)$ & .4300 & $(.1630)$ \\
\hline August $\left(\log _{10}+1\right)$ & .6814 & ${ }^{\mathrm{a}}(.0627)$ & -.0559 & $(.8953)$ & .0600 & $(.8530)$ & .5556 & ${ }^{\mathrm{a}}(.0607)$ \\
\hline September $\left(\log _{10}+1\right)$ & .8069 & $\mathrm{~b}(.0155)$ & .1210 & $(.7754)$ & .3116 & $(.3242)$ & .3560 & $(.2561)$ \\
\hline \multicolumn{9}{|c|}{ Magnitude and duration of annual extremes $\left(\mathrm{m}^{3} / \mathrm{sec}\right)$} \\
\hline 1-day minimum $\left(\log _{10}+1\right)$ & 0.4903 & $(0.2174)$ & 0.4054 & $(0.3191)$ & 0.2661 & $(0.4032)$ & 0.4401 & $(0.1522)$ \\
\hline 1-day maximum $\left(\log _{10}+1\right)$ & .5319 & $(.1748)$ & .1904 & $(.6516)$ & .2742 & $(.3885)$ & .4422 & $(.1500)$ \\
\hline 3-day minimum $\left(\log _{10}+1\right)$ & .4874 & $(.2206)$ & .4119 & $(.3106)$ & .2686 & $(.3987)$ & .4474 & $(.1447)$ \\
\hline 3-day maximum $\left(\log _{10}+1\right)$ & .5136 & $(.1929)$ & .1634 & $(.6990)$ & .2734 & $(.3899)$ & .4794 & $(.1148)$ \\
\hline 7-day minimum $\left(\log _{10}+1\right)$ & .4672 & $(.2432)$ & .4462 & $(.2678)$ & .2914 & $(.3581)$ & .4446 & $(.1476)$ \\
\hline 7-day maximum $\left(\log _{10}+1\right)$ & .5138 & $(.1928)$ & .1845 & $(.6618)$ & .3017 & $(.3405)$ & .4560 & $(.1363)$ \\
\hline 30-day minimum $\left(\log _{10}+1\right)$ & .4751 & $(.2341)$ & .4446 & $(.2697)$ & .3219 & $(.3075)$ & .4867 & $(.1086)$ \\
\hline 30-day maximum $\left(\log _{10}+1\right)$ & .4718 & $(.2379)$ & .1601 & $(.7049)$ & .2894 & $(.3616)$ & .5505 & $\mathrm{a}(.0636)$ \\
\hline 90-day minimum $\left(\log _{10}+1\right)$ & .5812 & $(.1308)$ & .3571 & $(.3852)$ & .1101 & $(.7335)$ & .4118 & $(.1835)$ \\
\hline 90-day maximum $\left(\log _{10}+1\right)$ & .4492 & $(.2642)$ & .1313 & $(.7566)$ & .2496 & $(.4339)$ & .6079 & ${ }^{b}(.0360)$ \\
\hline \multicolumn{9}{|c|}{ Timing of annual extremes (day of the year) } \\
\hline Annual minimum & 0.6124 & $(0.1065)$ & -0.0831 & $(0.8449)$ & -0.4004 & $(0.1972)$ & 0.1031 & $(0.7498)$ \\
\hline Annual maximum & .8109 & $\mathrm{~b}(.0146)$ & -.2668 & $(.5230)$ & -.0837 & $(.7960)$ & .3415 & $(.2773)$ \\
\hline \multicolumn{9}{|c|}{ Rate and frequency of change in conditions } \\
\hline Fall rate $\left(\mathrm{m}^{3} / \mathrm{sec}, \log _{10}+1\right)$ & 0.6420 & ${ }^{\mathrm{a}}(0.0861)$ & 0.1283 & $(0.7621)$ & 0.1310 & $(0.6849)$ & 0.4232 & $(0.1705)$ \\
\hline Rise rate $\left(\mathrm{m}^{3} / \mathrm{sec}, \log _{10}+1\right)$ & .6418 & ${ }^{\mathrm{a}}(.0862)$ & .2036 & $(.6288)$ & .2067 & $(.5191)$ & .6129 & ${ }^{b}(.0341)$ \\
\hline $\begin{array}{l}\text { Number of reversals between rising } \\
\text { and falling discharges }\end{array}$ & .3231 & $(.4350)$ & -.5113 & $(.1953)$ & -.4644 & $(.1282)$ & -.2322 & $(.4676)$ \\
\hline \multicolumn{9}{|c|}{ Frequency and duration of low and high pulses } \\
\hline Low-pulse length in days $\left(\log _{10}+1\right)$ & -0.6853 & $\mathrm{a}(0.0607)$ & -0.4290 & $(0.2889)$ & -0.2614 & $(0.4118)$ & 0.3668 & $(0.2408)$ \\
\hline Low-pulse count $\left(\log _{10}+1\right)$ & -.9645 & ${ }^{\mathrm{b}}(.0001)$ & .4977 & $(.2094)$ & .1664 & $(.6053)$ & -.6704 & ${ }^{\mathrm{b}}(.0170)$ \\
\hline High-pulse length in days & 6703 & a $(.0689)$ & .4429 & $(.2718)$ & .2835 & $(.3718)$ & .3063 & $(.3328)$ \\
\hline High-pulse count & .4456 & $(.2686)$ & .1255 & $(.7671)$ & -.0312 & $(.9233)$ & -.0255 & $(.9373)$ \\
\hline
\end{tabular}


Table 8. Site-level Pearson's correlation coefficients of Neosho madtom densities with the Indicators of Hydrologic Alteration parameters for the Spring River sites. Pearson's correlation coefficients and P-values are summarized for each site.-Continued

[The transformation in parentheses was performed on the indicated variables. MDC, Missouri Department of Conservation; $n$, number of years; $\mathrm{m}^{3} / \mathrm{sec}$, cubic meter per second; $\log _{10}$, logarithm with base 10$]$

\begin{tabular}{|c|c|c|c|c|}
\hline \multirow[b]{2}{*}{ Parameter } & \multicolumn{4}{|c|}{ Messer (n = 3) } \\
\hline & \multicolumn{2}{|c|}{$\begin{array}{l}\text { Coefficient from } \\
\text { preceding water year } \\
\text { (P-value) }\end{array}$} & \multicolumn{2}{|c|}{$\begin{array}{l}\text { Coefficient from } \\
\text { current water year } \\
\text { (P-value) }\end{array}$} \\
\hline \multicolumn{5}{|c|}{ Monthly discharge $\left(\mathrm{m}^{3} / \mathrm{sec}\right)$} \\
\hline October $\left(\log _{10}+1\right)$ & 0.8731 & $(0.3242)$ & -0.0194 & $(0.9877)$ \\
\hline November $\left(\log _{10}+1\right)$ & .2514 & $(.8382)$ & .3931 & $(.7428)$ \\
\hline December $\left(\log _{10}+1\right)$ & -.4904 & $(.6737)$ & -.8956 & $(.2934)$ \\
\hline January $\left(\log _{10}+1\right)$ & -.8635 & $(.3365)$ & .2128 & $(.8635)$ \\
\hline Feburary $\left(\log _{10}+1\right)$ & -.7494 & $(.4607)$ & -.2502 & $(.8390)$ \\
\hline $\operatorname{March}\left(\log _{10}+1\right)$ & -.7558 & $(.4545)$ & -.6476 & $(.5515)$ \\
\hline April $\left(\log _{10}+1\right)$ & .3695 & $(.7590)$ & -.0195 & $(.9876)$ \\
\hline May $\left(\log _{10}+1\right)$ & -.2497 & $(.8393)$ & .9123 & $(.2687)$ \\
\hline June $\left(\log _{10}+1\right)$ & -.6615 & $(.5398)$ & .9322 & $(.2359)$ \\
\hline July $\left(\log _{10}+1\right)$ & -.9583 & $(.1846)$ & .8492 & $(.3542)$ \\
\hline August $\left(\log _{10}+1\right)$ & -.8891 & $(.3026)$ & .9055 & $(.2790)$ \\
\hline September $\left(\log _{10}+1\right)$ & -.4692 & $(.6891)$ & .4052 & $(.7344)$ \\
\hline \multicolumn{5}{|c|}{ Magnitude and duration of annual extremes $\left(\mathrm{m}^{3} / \mathrm{sec}\right)$} \\
\hline 1-day minimum $\left(\log _{10}+1\right)$ & 0.0284 & $(0.9819)$ & 0.5231 & $(0.6495)$ \\
\hline 1-day maximum $\left(\log _{10}+1\right)$ & .0677 & $(.9569)$ & -.1869 & $(.8803)$ \\
\hline 3-day minimum $\left(\log _{10}+1\right)$ & .0353 & $(.9775)$ & .5280 & $(.6459)$ \\
\hline 3-day maximum $\left(\log _{10}+1\right)$ & .0556 & $(.9646)$ & .0453 & $(.9712)$ \\
\hline 7-day minimum $\left(\log _{10}+1\right)$ & .0984 & $(.9373)$ & .4810 & $(.6806)$ \\
\hline 7-day maximum $\left(\log _{10}+1\right)$ & .1354 & $(.9136)$ & -.0265 & $(.9831)$ \\
\hline 30-day minimum $\left(\log _{10}+1\right)$ & .0979 & $(.9376)$ & .5958 & $(.5937)$ \\
\hline 30-day maximum $\left(\log _{10}+1\right)$ & .2988 & $(.8068)$ & .5901 & $(.5981)$ \\
\hline 90-day minimum $\left(\log _{10}+1\right)$ & -.6799 & $(.5241)$ & 6920 & $(.5135)$ \\
\hline 90-day maximum $\left(\log _{10}+1\right)$ & .1134 & $(.9277)$ & .9712 & $(.1531)$ \\
\hline \multicolumn{5}{|c|}{ Timing of annual extremes (day of the year) } \\
\hline Annual minimum & -0.9974 & $\mathrm{~b}(0.0455)$ & 0.9200 & $(0.2563)$ \\
\hline Annual maximum & -.3406 & $(.7787)$ & .7907 & $(.4194)$ \\
\hline \multicolumn{5}{|c|}{ Rate and frequency of change in conditions } \\
\hline Fall rate $\left(\mathrm{m}^{3} / \mathrm{sec}, \log _{10}+1\right)$ & -0.7547 & $(0.4556)$ & 0.9966 & $\mathrm{a}(0.0529)$ \\
\hline Rise rate $\left(\mathrm{m}^{3} / \mathrm{sec}, \log _{10}+1\right)$ & -.5069 & $(.6616)$ & .8935 & $(.2965)$ \\
\hline $\begin{array}{l}\text { Number of reversals between rising } \\
\text { and falling discharges }\end{array}$ & -.9762 & $(.1391)$ & -.2250 & $(.8555)$ \\
\hline \multicolumn{5}{|c|}{ Frequency and duration of low and high pulses } \\
\hline Low-pulse length in days $\left(\log _{10}+1\right)$ & 0.3389 & $(0.7799)$ & 0.8523 & $(0.3504)$ \\
\hline Low-pulse count $\left(\log _{10}+1\right)$ & .7442 & $(.4656)$ & -1.0000 & $\mathrm{~b}(<.0001)$ \\
\hline High-pulse length in days & -.1628 & $(.8959)$ & -.8660 & $(.3333)$ \\
\hline High-pulse count & -.8660 & $(.3333)$ & -1.0000 & $\mathrm{~b}(<.0001)$ \\
\hline
\end{tabular}

${ }^{\text {a }}$ Indicates significance at the 0.10 level.

${ }^{\mathrm{b}}$ Indicates significance at the 0.05 level. 


\section{Summary}

At the locations monitored by the U.S. Fish and Wildlife Service, the density of Neosho madtoms has steadily declined until 2008; however, the associations of Neosho madtom densities with respect to the John Redmond Reservoir and other catfish species remains consistent with Wildhaber and others (2000). In the Neosho and Spring Rivers, Neosho madtoms had a significant positive association with all catfish species. The position of the site relative to the reservoir explained a significant amount of the variation only in the Neosho madtom density after accounting for the yearly variation. The average density of Neosho madtoms at the streamgage immediately below the reservoir had the second lowest compared to the other streamgages. The positive IHA associations with Neosho madtoms that remained consistent from the previous report of Wildhaber and others (2000) included the 1-, 3-, and 7-day minima and the annual minimum from the previous water year and the 1-, 3-, 7-, and 30-day minima from the current water year.

\section{Acknowledgments}

This study was jointly funded and undertaken by the U.S. Geological Survey Science Support Partnership Program and the U.S. Fish and Wildlife Service, through its Ecological Services Field Offices in Manhattan, Kans., and Tulsa, Okla. The authors thank Vernon Tabor and Dan Mulhern of the U.S. Fish and Wildlife Service for the collection of field data from Kansas and Oklahoma, and Doug Novinger of the Missouri Department of Conservation for the use of data collected from the Spring River in Missouri. The authors gratefully acknowledge the cooperation of the many private landowners in Kansas, Missouri, and Oklahoma who granted permission to sample on their property.

\section{References Cited}

Cross, F.B., and Collins, J.T., 1995, Fishes in Kansas, (2d ed.): Lawrence, Kans., University Press of Kansas, Public Education Series, 315 p.
Fuselier, Linda, and Edds, D.R., 1994, Seasonal variation in habitat use by the Neosho madtom (Teleostei-Ictaluridae-Noturus placidus): Southwestern Naturalist, v. 39, no. 3, p. 217-223.

Luttrell, G.R., Larson, R.D., Stark, W.J., Ashbaugh, N.A., Echelle, A.A., and Zale, A.V., 1992, Status and distribution of the Neosho madtom (Noturus placidus) in Oklahoma: Proceedings of the Oklahoma Academy of Science, v. 72, no. 1992, p. 5-6.

Milliken, G.G., and Johnson, D.E., 1984, Analysis of messy data, volume I-designed experiments: Belmont, Calif., Wadsworth, 473 p.

Moss, R.E., 1983, Microhabitat selection in Neosho River riffles: Lawrence, Kans., University of Kansas, Ph.D. dissertation, $294 \mathrm{p}$.

Nature Conservancy, 1996-2006, Indicators of hydrologic alteration: Haydon, Colo., Nature Conservancy and Smythe Scientific Software.

SAS, 2004, SAS for Windows 9.1.3 TS1M3: Cary, N.C., SAS Institute.

Taylor, W.R., 1969, A revision of the catfish genus Noturus Rafinesque, with an analysis of higher groups in the Ictaluridae: Washington, D.C., Smithsonian Institution Press, U.S. Museum Bulletin 282, 315 p.

U.S. Fish and Wildlife Service, 1991, Neosho madtom recovery plan: Denver, Colo., U.S. Fish and Wildlife Service, $42 \mathrm{p}$.

Wildhaber, M.L., Tabor, V.M., Whitaker, J.E., Allert, A.L., Mulhern, D.W., Lamberson, P.J., and Powell, K.L., 2000, Ictalurid populations in relation to the presence of a mainstem reservoir in a midwestern warmwater stream with emphasis on the threatened Neosho madtom: Transactions of the American Fisheries Society, v. 129, no. 6, p. 1,2641,280 .

Wilkinson, Chris, Edds, D.R., Dorlac, Joseph, Wildhaber, M.L., Schmitt, C.J., and Allert, Ann, 1996, Neosho madtom distribution and abundance in the Spring River: Southwestern Naturalist, v. 41, no. 1, p. 78-81. 
Appendix 
Table 1. Yearly median Indicators of Hydrologic Alteration parameters averaged over the years for the Cottonwood/Neosho River sites.

[n, number of years; NWA, National Wildlife Area; $\mathrm{m}^{3} / \mathrm{sec}$, cubic meter per second]

\begin{tabular}{|c|c|c|c|c|c|c|}
\hline \multirow{2}{*}{ Parameter } & \multicolumn{2}{|c|}{$\begin{array}{c}\text { Emporia } \\
(\mathbf{n}=\mathbf{8})\end{array}$} & \multicolumn{2}{|c|}{$\begin{array}{l}\text { Emporia location } 4 \\
\qquad(n=5)\end{array}$} & \multicolumn{2}{|c|}{$\begin{array}{l}\text { Humbolt } \\
\text { (n=10) }\end{array}$} \\
\hline & $\begin{array}{l}\text { Preceeding } \\
\text { water year }\end{array}$ & $\begin{array}{c}\text { Current } \\
\text { water year }\end{array}$ & $\begin{array}{l}\text { Preceeding } \\
\text { water year }\end{array}$ & $\begin{array}{c}\text { Current } \\
\text { water year }\end{array}$ & $\begin{array}{l}\text { Preceeding } \\
\text { water year }\end{array}$ & $\begin{array}{c}\text { Current } \\
\text { water year }\end{array}$ \\
\hline \multicolumn{7}{|c|}{ Monthly discharge $\left(\mathrm{m}^{3} / \mathrm{sec}\right)$} \\
\hline October & 32.19 & 54.14 & 168.17 & 201.08 & 198.01 & 173.93 \\
\hline November & 60.98 & 77.13 & 364.26 & 245.30 & 285.21 & 181.56 \\
\hline December & 57.84 & 54.53 & 324.26 & 275.93 & 312.40 & 276.48 \\
\hline January & 45.43 & 46.13 & 249.34 & 226.48 & 295.08 & 431.50 \\
\hline Feburary & 46.40 & 48.60 & 258.97 & 210.00 & 430.84 & 544.07 \\
\hline March & 95.14 & 80.80 & 410.22 & 402.31 & 720.35 & $1,108.55$ \\
\hline April & 158.97 & 153.63 & 552.93 & 462.08 & $1,304.06$ & $1,235.75$ \\
\hline May & 458.39 & 332.61 & $1,964.64$ & $1,470.06$ & $3,006.31$ & $1,892.89$ \\
\hline June & 170.56 & 175.87 & $1,072.30$ & 824.91 & $2,891.64$ & $1,562.53$ \\
\hline July & 75.64 & 91.08 & 639.19 & 389.21 & 891.71 & $1,000.69$ \\
\hline August & 63.77 & 55.10 & 417.65 & 280.90 & 347.46 & 321.36 \\
\hline September & 35.14 & 28.43 & 232.36 & 163.93 & 145.74 & 300.44 \\
\hline \multicolumn{7}{|c|}{ Magnitude and duration of annual extremes $\left(\mathrm{m}^{3} / \mathrm{sec}\right)$} \\
\hline 1-day minimum & 8.43 & 9.90 & 70.01 & 64.90 & 31.69 & 32.89 \\
\hline 1-day maximum & $4,734.31$ & $4,687.96$ & $19,558.14$ & $15,158.95$ & $19,564.61$ & $18,691.73$ \\
\hline 3-day minimum & 10.27 & 10.39 & 72.12 & 66.70 & 35.25 & 36.47 \\
\hline 3-day maximum & $3,370.96$ & $3,427.49$ & $16,358.28$ & $12,628.94$ & $14,395.82$ & $14,417.32$ \\
\hline 7-day minimum & 12.07 & 12.59 & 77.86 & 71.30 & 38.81 & 39.37 \\
\hline 7-day maximum & $2,429.74$ & $2,437.07$ & $11,307.25$ & $8,947.87$ & $10,681.09$ & $10,372.45$ \\
\hline 30-day minimum & 17.68 & 18.30 & 98.41 & 87.49 & 65.86 & 66.68 \\
\hline 30-day maximum & $1,200.03$ & $1,236.12$ & $4,885.10$ & $3,938.18$ & $6,984.55$ & $6,755.03$ \\
\hline 90-day minimum & 49.36 & 43.61 & 285.28 & 222.54 & 239.85 & 214.30 \\
\hline 90-day maximum & 684.59 & 688.61 & $2,799.13$ & $2,183.48$ & $4,307.81$ & $3,912.84$ \\
\hline \multicolumn{7}{|c|}{ Timing of annual extremes (day of the year) } \\
\hline Annual minimum & 247.00 & 249.38 & 255.00 & 250.00 & 292.70 & 278.50 \\
\hline Annual maximum & 137.25 & 156.50 & 148.00 & 137.60 & 176.30 & 175.50 \\
\hline \multicolumn{7}{|c|}{ Rate and frequency of change in conditions } \\
\hline Fall rate $\left(\mathrm{m}^{3} / \mathrm{sec}\right)$ & 7.63 & 6.70 & 30.43 & 21.70 & 48.59 & 49.93 \\
\hline Rise rate $\left(\mathrm{m}^{3} / \mathrm{sec}\right)$ & 12.02 & 11.49 & 37.45 & 30.05 & 75.54 & 85.34 \\
\hline $\begin{array}{l}\text { Number of reversals between } \\
\text { rising and falling discharges }\end{array}$ & 108.63 & 106.50 & 105.60 & 100.60 & 116.50 & 109.30 \\
\hline \multicolumn{7}{|c|}{ Frequency and duration of low and high pulses } \\
\hline Low-pulse length in days & 6.19 & 5.99 & 4.45 & 6.97 & 4.97 & 6.65 \\
\hline Low-pulse count & 4.44 & 4.86 & 2.47 & 2.47 & 3.75 & 3.88 \\
\hline High-pulse length in days & 3.94 & 4.25 & 5.30 & 4.90 & 5.50 & 4.05 \\
\hline High-pulse count & 11.00 & 10.38 & 9.20 & 10.40 & 10.40 & 9.70 \\
\hline
\end{tabular}


Table 1. Yearly median Indicators of Hydrologic Alteration parameters averaged over the years for the Cottonwood/Neosho River sites.-Continued

[n, number of years; NWA, National Wildlife Area; $\mathrm{m}^{3} / \mathrm{sec}$, cubic meter per second]

\begin{tabular}{|c|c|c|c|c|c|c|}
\hline \multirow{2}{*}{ Parameter } & \multicolumn{2}{|c|}{$\begin{array}{l}\text { NWA } \\
(n=12)\end{array}$} & \multicolumn{2}{|c|}{$\begin{array}{l}\text { Oswego } \\
(n=13)\end{array}$} & \multicolumn{2}{|c|}{$\begin{array}{c}\text { Oklahoma } \\
(n=9)\end{array}$} \\
\hline & $\begin{array}{l}\text { Preceeding } \\
\text { water year }\end{array}$ & $\begin{array}{c}\text { Current } \\
\text { water year }\end{array}$ & $\begin{array}{l}\text { Preceeding } \\
\text { water year }\end{array}$ & $\begin{array}{c}\text { Current } \\
\text { water year }\end{array}$ & $\begin{array}{l}\text { Preceeding } \\
\text { water year }\end{array}$ & $\begin{array}{c}\text { Current } \\
\text { water year }\end{array}$ \\
\hline \multicolumn{7}{|c|}{ Monthly discharge $\left(\mathrm{m}^{3} / \mathrm{sec}\right)$} \\
\hline October & 233.56 & 217.81 & 223.03 & 160.75 & 290.56 & 280.36 \\
\hline November & 309.94 & 307.39 & 237.65 & 256.89 & 262.67 & 427.87 \\
\hline December & 629.10 & 487.99 & 437.47 & 390.91 & 547.26 & 763.86 \\
\hline January & 513.20 & 496.84 & 409.71 & 367.24 & 462.50 & 643.90 \\
\hline Feburary & 691.66 & 849.80 & 610.18 & 565.43 & 717.19 & $1,073.12$ \\
\hline March & $1,209.07$ & $1,889.49$ & $1,353.92$ & $1,357.08$ & $2,063.01$ & $2,864.93$ \\
\hline April & $1,663.87$ & $1,924.16$ & $1,292.30$ & $1,368.54$ & $1,458.01$ & $1,991.75$ \\
\hline May & $3,846.54$ & $3,185.23$ & $2,943.11$ & $2,513.74$ & $3,311.38$ & $3,463.61$ \\
\hline June & $3,377.17$ & $2,816.26$ & $2,955.25$ & $2,982.97$ & $4,142.43$ & $4,643.51$ \\
\hline July & $1,407.36$ & $1,191.46$ & 737.97 & $1,158.14$ & $1,158.88$ & $1,577.99$ \\
\hline August & 372.97 & 351.66 & 259.71 & 309.59 & 257.17 & 305.36 \\
\hline September & 356.54 & 405.30 & 243.99 & 264.79 & 388.70 & 435.97 \\
\hline \multicolumn{7}{|c|}{ Magnitude and duration of annual extremes $\left(\mathrm{m}^{3} / \mathrm{sec}\right)$} \\
\hline 1-day minimum & 39.85 & 43.22 & 35.79 & 31.32 & 42.54 & 46.42 \\
\hline 1-day maximum & $31,598.66$ & $26,697.08$ & $25,837.65$ & $26,367.16$ & $29,354.24$ & $32,309.23$ \\
\hline 3-day minimum & 42.37 & 45.67 & 37.53 & 33.25 & 44.40 & 48.60 \\
\hline 3-day maximum & $25,924.96$ & $22,371.97$ & $21,170.99$ & $21,850.22$ & $26,185.35$ & $29,034.32$ \\
\hline 7-day minimum & 47.90 & 50.24 & 42.09 & 37.26 & 52.48 & 57.84 \\
\hline 7-day maximum & $19,325.74$ & $16,968.41$ & $15,354.16$ & $15,958.28$ & $19,655.98$ & $21,975.09$ \\
\hline 30-day minimum & 92.81 & 91.37 & 80.46 & 72.62 & 93.69 & 115.86 \\
\hline 30-day maximum & $10,687.98$ & $10,134.23$ & $8,842.86$ & $9,713.38$ & $10,828.65$ & $12,387.71$ \\
\hline 90-day minimum & 411.79 & 369.09 & 327.01 & 326.76 & 460.46 & 696.13 \\
\hline 90-day maximum & $6,283.51$ & $5,824.69$ & $5,039.99$ & $5,520.31$ & $6,442.82$ & $7,322.51$ \\
\hline \multicolumn{7}{|c|}{ Timing of annual extremes (day of the year) } \\
\hline Annual minimum & 280.83 & 270.08 & 278.85 & 277.62 & 271.22 & 264.67 \\
\hline Annual maximum & 177.25 & 166.08 & 163.69 & 160.54 & 122.33 & 105.33 \\
\hline \multicolumn{7}{|c|}{ Rate and frequency of change in conditions } \\
\hline Fall rate $\left(\mathrm{m}^{3} / \mathrm{sec}\right)$ & 82.07 & 82.62 & 63.04 & 64.76 & 82.51 & 107.81 \\
\hline Rise rate $\left(\mathrm{m}^{3} / \mathrm{sec}\right)$ & 151.12 & 157.57 & 118.02 & 130.17 & 191.18 & 246.40 \\
\hline $\begin{array}{l}\text { Number of reversals between } \\
\text { rising and falling discharges }\end{array}$ & 98.42 & 100.25 & 98.00 & 96.00 & 98.33 & 97.44 \\
\hline \multicolumn{7}{|c|}{ Frequency and duration of low and high pulses } \\
\hline Low-pulse length in days & 8.58 & 7.56 & 10.10 & 7.81 & 7.11 & 7.82 \\
\hline Low-pulse count & 2.94 & 2.38 & 3.31 & 2.64 & 4.66 & 3.90 \\
\hline High-pulse length in days & 3.75 & 4.04 & 3.58 & 3.62 & 4.28 & 4.39 \\
\hline High-pulse count & 11.17 & 11.25 & 10.38 & 10.15 & 11.44 & 11.78 \\
\hline
\end{tabular}


Table 2. Yearly median Indicators of Hydrologic Alteration parameters averaged over the years for the Spring River sites.

[Values were back-transformed if transformation was required for the analysis. MDC, Missouri Department of Conservation; $\mathrm{n}$, number of years; $\mathrm{m}^{3} / \mathrm{sec}$, cubic meter per second]

\begin{tabular}{|c|c|c|c|c|c|c|}
\hline \multirow{2}{*}{ Parameter } & \multicolumn{2}{|c|}{$\begin{array}{l}\text { MDC } \\
(n=8)\end{array}$} & \multicolumn{2}{|c|}{$\begin{array}{l}\text { Spring } \\
(n=12)\end{array}$} & \multicolumn{2}{|c|}{$\begin{array}{c}\text { Messer } \\
(n=3)\end{array}$} \\
\hline & $\begin{array}{l}\text { Preceeding } \\
\text { water year }\end{array}$ & $\begin{array}{c}\text { Current } \\
\text { water year }\end{array}$ & $\begin{array}{l}\text { Preceeding } \\
\text { water year }\end{array}$ & $\begin{array}{c}\text { Current } \\
\text { water year }\end{array}$ & $\begin{array}{l}\text { Preceeding } \\
\text { water year }\end{array}$ & $\begin{array}{c}\text { Current } \\
\text { water year }\end{array}$ \\
\hline \multicolumn{7}{|c|}{ Monthly discharge $\left(\mathrm{m}^{3} / \mathrm{sec}\right)$} \\
\hline October & 106.74 & 112.32 & 153.58 & 268.88 & 211.28 & 147.54 \\
\hline November & 226.93 & 207.64 & 279.97 & $1,342.99$ & 466.43 & 267.82 \\
\hline December & 331.27 & 306.33 & 374.88 & 755.67 & 942.66 & 332.03 \\
\hline January & 337.78 & 356.49 & 368.80 & 869.57 & 750.81 & 337.82 \\
\hline Feburary & 360.35 & 389.80 & 420.95 & 671.10 & 631.70 & 373.91 \\
\hline March & 463.37 & 466.33 & 659.30 & 618.43 & $1,030.28$ & 579.36 \\
\hline April & 523.54 & 641.78 & 529.29 & 871.35 & $1,094.63$ & 485.07 \\
\hline May & 873.59 & 979.88 & 720.27 & $1,306.32$ & $1,145.61$ & 757.11 \\
\hline June & 531.19 & 654.00 & 504.70 & 573.42 & 882.21 & 475.53 \\
\hline July & 263.00 & 262.84 & 277.35 & 196.65 & 590.45 & 235.95 \\
\hline August & 117.26 & 123.00 & 121.45 & 128.31 & 217.76 & 110.59 \\
\hline September & 140.16 & 142.91 & 113.71 & 106.16 & 245.24 & 96.87 \\
\hline \multicolumn{7}{|c|}{ Magnitude and duration of annual extremes $\left(\mathrm{m}^{3} / \mathrm{sec}\right)$} \\
\hline 1-day minimum & 44.84 & 44.07 & 59.30 & 66.21 & 89.97 & 53.94 \\
\hline 1-day maximum & $20,325.69$ & $17,735.08$ & $19,728.91$ & $32,748.74$ & $45,174.74$ & $15,465.45$ \\
\hline 3-day minimum & 46.59 & 45.84 & 61.19 & 68.56 & 91.82 & 55.85 \\
\hline 3-day maximum & $15,394.38$ & $13,737.79$ & $14,717.90$ & $27,078.45$ & $34,096.48$ & $11,983.55$ \\
\hline 7-day minimum & 49.98 & 48.98 & 65.10 & 75.24 & 95.26 & 59.31 \\
\hline 7-day maximum & $9,292.88$ & $8,154.82$ & $8,875.81$ & $15,243.65$ & $18,688.64$ & $7,399.98$ \\
\hline 30-day minimum & 63.94 & 62.64 & 84.11 & 98.39 & 114.78 & 77.27 \\
\hline 30-day maximum & $3,622.62$ & $3,671.54$ & $3,696.22$ & $6,530.44$ & $6,222.79$ & $3,236.96$ \\
\hline 90-day minimum & 158.07 & 152.43 & 230.57 & 204.39 & 537.47 & 194.57 \\
\hline 90-day maximum & $1,992.59$ & $2,251.90$ & $1,990.66$ & $3,689.38$ & $3,211.96$ & $1,822.68$ \\
\hline \multicolumn{7}{|c|}{ Timing of annual extremes (day of the year) } \\
\hline Annual minimum & 269.63 & 275.75 & 272.92 & 259.67 & 282.33 & 271.08 \\
\hline Annual maximum & 125.75 & 109.00 & 125.42 & 90.67 & 145.67 & 117.50 \\
\hline \multicolumn{7}{|c|}{ Rate and frequency of change in conditions } \\
\hline Fall rate $\left(\mathrm{m}^{3} / \mathrm{sec}\right)$ & 22.75 & 21.01 & 21.71 & 19.17 & 47.62 & 27.04 \\
\hline Rise rate $\left(\mathrm{m}^{3} / \mathrm{sec}\right)$ & 52.71 & 66.85 & 63.61 & 116.44 & 212.20 & 56.20 \\
\hline $\begin{array}{l}\text { Number of reversals between } \\
\text { rising and falling discharges }\end{array}$ & 84.25 & 83.38 & 81.58 & 76.67 & 81.00 & 81.25 \\
\hline \multicolumn{7}{|c|}{ Frequency and duration of low and high pulses } \\
\hline Low-pulse length in days & 16.48 & 23.70 & 13.67 & 33.14 & 4.34 & 22.52 \\
\hline Low-pulse count & 2.12 & 2.40 & 2.67 & 2.68 & 1.47 & 2.97 \\
\hline High-pulse length in days & 4.75 & 4.19 & 4.21 & 5.00 & 6.67 & 3.63 \\
\hline High-pulse count & 10.75 & 9.63 & 11.42 & 9.00 & 14.00 & 10.58 \\
\hline
\end{tabular}


Publishing support provided by:

Rolla Publishing Service Center

For more information concerning this publication, contact:

Director, USGS Columbia Environmental Research Center

4200 New Haven Road

Columbia, MO 65201

(573) 875-5399

Or visit the Columbia Environmental Research Center Web site at: http://www.cerc.usgs.gov 


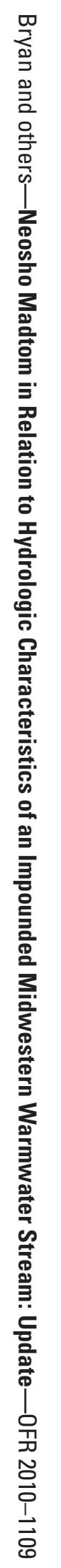

